\title{
FROM PERIODIC TRAVELLING WAVES TO SOLITONS OF A 2D WATER WAVE SYSTEM*
}

\author{
JOSÉ R. QUINTERO ${ }^{\dagger}$
}

\begin{abstract}
We use a variational approach to establish the existence of $x$-periodic travelling waves and its interrelation with solitons for a $2 \mathrm{D}$ water wave system for three-dimensional water wave dynamics in the weakly nonlinear long-wave regime. As common in many $1 \mathrm{D}$ water wave models, we show that a special sequence of the $x$-periodic $2 \mathrm{D}$ travelling wave solutions parametrized by the period $k$ is uniformly bounded in norm and converges to a soliton in $\mathbb{R}^{2}$ (solitary wave of finite energy) in an appropriate sense, indicating that the shape of $x$-periodic $2 \mathrm{D}$ travelling waves of period $k$ and solitons are almost the same, as the period $k$ is big enough.
\end{abstract}

Key words. 2D Boussinesq water wave system, Periodic travelling waves, solitons, variational methods.

AMS subject classifications. 35Q35, 37K40, 35B10, 35A15.

1. Introduction. J. Quintero in [1] showed that the evolution of three dimensional long water waves with small amplitude can be reduced to studying solutions of the 2D Boussinesq type system,

$$
\left\{\begin{aligned}
\eta_{t}+\epsilon \nabla \cdot\left(\eta\left(\Phi_{x}^{p}, \Phi_{y}^{p}\right)\right)+\Delta \Phi-\frac{\mu}{6} \Delta^{2} \Phi & =0 \\
\Phi_{t}+\eta-\mu\left(\sigma-\frac{1}{2}\right) \Delta \eta+\frac{\epsilon}{p+1}\left(\Phi_{x}^{p+1}+\Phi_{y}^{p+1}\right) & =0
\end{aligned}\right.
$$

where $\epsilon$ is the amplitude parameter (nonlinearity coefficient), $\mu=\left(h_{0} / L\right)^{2}$ is the long-wave parameter (dispersion coefficient), $\sigma^{-1}$ is the Bond number (associated with the surface tension), and $p=1$. The variable $\Phi$ is the rescale nondimensional velocity potential on the bottom $z=0$, and the variable $\eta$ is the rescaled free surface elevation (see [1]). For $0<|c|<1$ and $\sigma>\frac{1}{2}$, J. Quintero proved existence of solitons and also showed that in an appropriate scaling there are sequences of solitons that converge as $\epsilon, \mu \rightarrow 0$ to a soliton (after a translation) of the generalized KadomtsevPetviashivili equation (GKP-I) equation, meaning that this system has physically meaningful solitons (see [1]). On the other hand, J. Quintero proved the well possedness for the Cauchy problem and for $0<|c|<1$ and $\sigma>\frac{1}{2}$ orbital stability of solitons (see $[3])$.

The Boussinesq system considered in this paper has a close relationship with the generalized Benney-Luke equation and the generalized Kadomtsev-Petviashivili in the sense that the later models can be derived from the Boussinesq type system (1.1). Regarding the generalized Kadomtsev-Petviashivili and the Benney-Luke model, A. Pankov and K. Pflüger in [2] and J. Quintero in [4] analyzed the limit behavior of such $x$-periodic travelling solutions as the period $k$ goes to $+\infty$, proving, as done for some 1D model (see [13], [14], [15] ), that the shape of the $x$-periodic travelling waves (ground states) of period $k$ and solitary ground states are almost the same provided that the period is big enough, respectively. It is worth to mention that the Boussinesq system (1.1) is contained in a more general class of systems known as of

\footnotetext{
*Received March 4, 2013; accepted for publication October 4, 2013.

†Departamento Matemáticas, Universidad del Valle, A. A. 25360, Cali, Colombia (jose.quintero@ correounivalle.edu.co).
} 
Bona-Chen-Saut systems of the form

$$
\left\{\begin{aligned}
\eta_{t}+\nabla \cdot V+\epsilon\left(\nabla \cdot(\eta V)+e \Delta \nabla \cdot V-d \Delta \eta_{t}\right) & =0 \\
V_{t}+\nabla \eta+\epsilon\left(\frac{1}{2} \nabla|V|^{2}+a \Delta \nabla \eta-d \Delta V_{t}\right) & =0
\end{aligned}\right.
$$

where $a+b+e+d=\frac{1}{3}-\sigma$ with $a, e<0$ and $b, d \geq 0$, in the case of non zero surface tension (see [6], [7], [8], [10])). If we set $p=1, \epsilon=\mu, a=-\frac{1}{6}, e=\frac{1}{2}-\sigma, b=d=0$ and take $V=\nabla \Phi$, we see that the first two equations of the (1.2) system corresponds to the Boussinesq system considered in this work. J. Bona et. al. in [8] established rigorous convergence results in order to show that complete free-surface solutions for the Euler equations tend to associated solutions of these systems as the amplitude becomes small and the wavelength large, providing also a rigorous justification for many 2D water wave systems. In the one dimensional case, Chen et. al. in [11] and [12] established existence and orbital stability of travelling solutions for the Boussinesq system (1.2) for $\sigma>\frac{1}{3}, b=d>0$ and $a e=d^{2}$ in [11], and existence of travelling solutions (1.2) for $\sigma>\frac{1}{3}, b=d \geq 0$ in [12]. An interesting review in the case of existence of periodic 2D travelling waves for the full Euler equations (doubly periodic or periodic in one direction) appears in the work of M. Groves [9].

In this paper, we consider the existence of non trivial $x$-periodic travelling wave of period $k>0$ for the Boussinesq type system (1.1) for wave speed $0<|c|<1$ and $\sigma>\frac{1}{2}$, and also analyze the interrelation for the sequence of $x$-periodic travelling waves of period $k$ with solitons for this system, as $k \rightarrow \infty$. We note that the assumption on $\sigma$ are consistent with those used for the Boussinesq type system (1.2), where $a=-\frac{1}{6}$, $e=\frac{1}{2}-\sigma<0, b=d=0$, and $a+b+e+d=\frac{1}{3}-\sigma<0$.

The paper is organized as follows: In section 2. for $\epsilon>0, \mu>0, \sigma>\frac{1}{2}$ and $0<|c|<1$, we prove the existence of a $x$-periodic travelling of weak solutions of period $k$ for the Boussinesq type system (1.1). Periodic travelling waves correspond to critical points of the action functional $J_{c, k}$, for which the existence of critical points $U_{k}$ follows as a consequence of the Mountain Pass Theorem without the Palais-Smale condition. We also establish some local embedding results from the space of weak $x$-periodic travelling solutions (denoted by $X_{k}$ ) to a $L_{k}^{q}$ ( $x$-periodic of period $k$ ) type space and the corresponding local embedding result from the space of weak travelling solutions (denoted by $X$ ) to a $L^{q}\left(\mathbb{R}^{2}\right)$ type space. In order to achieve this goal, we build an extension operator $E_{k}$ from the space of $k$ periodic functions $X_{k}$ to the soliton space $X$. One of the main ingredient to establish the existence of periodic travelling waves is to characterized "vanishing" sequences in $X_{k}$ and $X$. We establish uniform bounds for Mountain Pass minimax value $\mathcal{J}_{k}(c)$ with respect to the period $k$. In section 3. from results in Section 2, weak $x$-periodic travelling waves solution of period $\mathrm{k}$ (denoted by $U_{k}$ ) of the generalized Boussinesq system for $k \geq 1$ are characterized as a minimizer of the minimax value $\mathcal{J}_{k}(c)$. We see that after an appropriate translation, there is a convergent subsequence in $X$ of the extended sequence $\left\{E_{k}\left(U_{k}\right)\right\}_{k}$, whose limit $U_{0}$ is a non trivial critical point of the functional $J_{c}$, with the property of being a weak solution of the Boussinesq system and a minimizer for $J_{c}$ (a soliton or a ground state) for the Boussinesq system (1.1). Finally, we establish the interrelation between $x$-periodic weak travelling wave solutions (ground states) of period $k$ and weak travelling wave solutions (solitons). In particular, we show that for a sequence $\left(U_{k}\right)_{k}$ of $k$-periodic travelling waves solutions of the Boussinesq type system (1.1) with $U_{k} \in X_{k}$ that there is a non trivial soliton $U_{0} \in X$ of the Boussinesq type system (1.1), 
and there is a sequence $\zeta_{k} \in \mathbb{R}^{2}$, we have that

$$
\lim _{k \rightarrow \infty}\left\|U_{k}-U_{0}\left(\cdot-\zeta_{k}\right)\right\|_{x_{k}} \rightarrow 0
$$

2. Periodic travelling waves for $0<|c|<1$ and $\sigma>\frac{1}{2}$. In this section for fixed $k>0$, we will establish the existence of a $x$-periodic travelling of period $k$ for the Boussinesq type system (1.1) for fixed positive values of the parameters $\epsilon$ and $\mu$, for non dimensional speed $0<|c|<1$, and for the surface tension coefficient in the range $\sigma>\frac{1}{2}$. We will see that periodic travelling waves are characterized as critical points of some functional (action), for which the existence of critical follows as a consequence of the Mountain Pass Theorem without the Palais-Smale condition and the existence of the local compactness property Lemma (2.3) (a local compact embedding result). Note that the parameters $\epsilon$ and $\mu$ can be scale from the equations when searching for travelling waves solutions. In fact, we look for travelling waves of the form

$$
\eta(x, y, t)=\left(\frac{1}{\epsilon^{1 / p}}\right) u\left(\frac{x-c t}{\sqrt{\mu}}, \frac{y}{\sqrt{\mu}}\right), \Phi(x, y, t)=\left(\frac{\sqrt{\mu}}{\epsilon^{1 / p}}\right) v\left(\frac{x-c t}{\sqrt{\mu}}, \frac{y}{\sqrt{\mu}}\right) \text {. }
$$

In other words, travelling-wave profile $(u, v)$ should satisfy the system

$$
\left(\begin{array}{c}
\Delta v-\frac{1}{6} \Delta^{2} v+\nabla \cdot\left(u\left(v_{x}^{p}, v_{y}^{p}\right)\right)-c u_{x} \\
u-\left(\sigma-\frac{1}{2}\right) \Delta u+\frac{1}{p+1}\left(v_{x}^{p+1}+v_{y}^{p+1}\right)-c v_{x}
\end{array}\right)=\left(\begin{array}{c}
0 \\
0
\end{array}\right)
$$

In order to determine the appropriate space to look for travelling waves, we find that the energy functional $\mathcal{E}$ associated with this system is given by

$$
\mathcal{E}(u, v)=\frac{1}{2} \int_{Q_{k}}\left[|\nabla v|^{2}+u^{2}+\frac{1}{6}|\Delta v|^{2}+\left(\sigma-\frac{1}{2}\right)|\nabla u|^{2}+\frac{2}{p+1} u\left(v_{x}^{p+1}+v_{y}^{p+1}\right)\right] d y d x,
$$

where $Q_{k}=\left[-\frac{k}{2}, \frac{k}{2}\right] \times \mathbb{R}$. So, the energy functional $\mathcal{E}$ is well defined if $u, u_{x}, u_{y}, v_{x}$, $v_{y}, v_{x x}, v_{x y}, v_{y y}$ belong to the space $L_{k}^{2}\left(\mathbb{R}^{2}\right)$, where

$L_{k}^{q}\left(\mathbb{R}^{2}\right)=\left\{f: \mathbb{R}^{2} \rightarrow \mathbb{R}: f \in L^{q}\left(Q_{k}\right)\right.$ and $x$-periodic of period $\left.k\right\},\|u\|_{L_{k}^{q}\left(\mathbb{R}^{2}\right)}=\|u\|_{L^{q}\left(Q_{k}\right)}$.

In inequalities below, $C$ denotes a generic constant whose value may change from instance to instance. Hereafter $p=\frac{p_{1}}{p_{2}}$ with $p_{i}$ being odd positive integer and $\operatorname{gcd}\left(p_{1}, p_{2}\right)=1$.

2.1. Preliminaries. We define the natural spaces in order to look at $x$-periodic weak solution period $k$ for the equation (2.4).

Definition 2.1. Let $C_{\text {per }}^{\infty}\left(\mathbb{R}^{2}\right)$ be the space of smooth functions which are $x$ periodic with period $k$ and have compact support in $y$ and define

$$
y_{k}=\left\{\left.\varphi\right|_{Q_{k}}: \varphi \in C_{p e r}^{\infty}\left(\mathbb{R}^{2}\right)\right\},
$$

Let $\mathcal{V}_{k}$ denote the closure of $y_{k}$ with respect to the norm given by

$$
\begin{aligned}
\|\phi\|_{\mathcal{V}_{k}}^{2} & :=\int_{Q_{k}}\left(\phi_{x}^{2}+\phi_{y}^{2}+\phi_{x x}^{2}+2 \phi_{x y}^{2}+\phi_{y y}^{2}\right) d y d x \\
& =\left\|\partial_{x} \phi\right\|_{H^{1}\left(Q_{k}\right)}^{2}+\left\|\partial_{y} \phi\right\|_{H^{1}\left(Q_{k}\right)}^{2} .
\end{aligned}
$$

Note that $\left(\mathcal{V}_{k},\|\cdot\|_{\mathcal{V}(k)}\right)$ is a Hilbert space with inner product

$$
(u, v)_{v_{k}}=\left(\partial_{x} u, \partial_{x} v\right)_{H_{k}^{1}\left(\mathbb{R}^{2}\right)}+\left(\partial_{y} u, \partial_{y} v\right)_{H_{k}^{1}\left(\mathbb{R}^{2}\right)},
$$


where $H_{k}^{1}\left(\mathbb{R}^{2}\right)$ is the Hilbert space of functions $u \in L_{k}^{2}\left(\mathbb{R}^{2}\right)$ such that $u_{x}, u_{y} \in L_{k}^{2}\left(\mathbb{R}^{2}\right)$. The space $H_{k}^{1}\left(\mathbb{R}^{2}\right)$ has the inner product given by

$$
(\phi, \phi)_{H_{k}^{1}\left(\mathbb{R}^{2}\right)}=(\phi, \phi)_{L^{2}\left(Q_{k}\right)}+\left(\partial_{x} \phi, \partial_{x} \phi\right)_{L^{2}\left(Q_{k}\right)}+\left(\partial_{y} \phi, \partial_{y} \phi\right)_{L^{2}\left(Q_{k}\right)} .
$$

In the case of solitons, the appropriate space, denoted by $\mathcal{V}$, is defined as the closure of $C_{0}^{\infty}\left(\mathbb{R}^{2}\right)$ with respect to the norm

$$
\begin{aligned}
\|\phi\|_{\mathcal{V}}^{2} & :=\int_{\mathbb{R}^{2}} \phi_{x}^{2}+\phi_{y}^{2}+\phi_{x x}^{2}+2 \phi_{x y}^{2}+\phi_{y y}^{2} d y d x \\
& =\left\|\partial_{x} \phi\right\|_{H^{1}\left(\mathbb{R}^{2}\right)}^{2}+\left\|\partial_{y} \phi\right\|_{H^{1}\left(\mathbb{R}^{2}\right)}^{2} .
\end{aligned}
$$

Embedding. Before we go further, we will establish a basic local embedding defined on $H_{k}^{1}\left(\mathbb{R}^{2}\right) \times \mathcal{V}_{k}$, which depends on the existence of an extension operator from $H_{k}^{1}\left(\mathbb{R}^{2}\right) \times \mathcal{V}_{k}$ to $H^{1}\left(\mathbb{R}^{2}\right) \times \mathcal{V}$. This type of extension was proved by J. Quintero for the Benney-Luke equation in [4] (see also A. Pankov and K. Pflüger in [2] for the (GKP) case). For $q \geq 2$ and $Q \subset \mathbb{R}^{2}$ and define the Banach space

$$
\mathcal{M}^{(q)}(Q): \overline{\left\langle C_{0}^{\infty}(Q),\|\cdot\|_{(q)}\right\rangle}, \quad\|\phi\|_{(q)}^{q}=\left\|\partial_{x} \phi\right\|_{L^{q}(Q)}^{q}+\left\|\partial_{y} \phi\right\|_{L^{q}(Q)}^{q} .
$$

Hereafter, we will use the following notation: $X=H^{1}\left(\mathbb{R}^{2}\right) \times \mathcal{V}, x_{k}=H_{k}^{1}\left(\mathbb{R}^{2}\right) \times \mathcal{V}_{k}$, and for $Q \subset \mathbb{R}^{2}$,

$$
\|\phi\|_{\mathcal{V}(Q)}^{2}:=\int_{Q}\left(\phi_{x}^{2}+\phi_{y}^{2}+\phi_{x x}^{2}+2 \phi_{x y}^{2}+\phi_{y y}^{2}\right) d y d x .
$$

Then we have the following embedding result

Lemma 2.1. (J. Quintero [4]) For $q \geq 2$, we have that

1. The embeddings $H^{1}\left(\mathbb{R}^{2}\right) \hookrightarrow L^{q}\left(\mathbb{R}^{2}\right)$ and $\mathcal{V} \hookrightarrow \mathcal{M}^{(q)}\left(\mathbb{R}^{2}\right)$ are continuous,

2. The embeddings $H^{1}\left(\mathbb{R}^{2}\right) \hookrightarrow L_{\text {loc }}^{q}\left(\mathbb{R}^{2}\right)$ and $\mathcal{V} \hookrightarrow \mathcal{M}_{\text {loc }}^{(q)}\left(\mathbb{R}^{2}\right)$ are compact.

3. The embedding $X \hookrightarrow L_{\text {loc }}^{q}\left(\mathbb{R}^{2}\right) \times \mathcal{M}_{\text {loc }}^{(q)}\left(\mathbb{R}^{2}\right)$ is compact,

where $f \in L_{l o c}^{q}\left(\mathbb{R}^{2}\right)$, if $f \in L^{q}(D)$ for any compact ball $D \in \mathbb{R}^{2}$. Clearly, $f \in \mathcal{M}_{\text {loc }}^{(q)}$, if $f_{x}, f_{y} \in L_{l o c}^{q}\left(\mathbb{R}^{2}\right)$.

In the case of the space $X_{k}$ we have a similar result. To do this, we require the use of a cut-off operator to extend any function in $\mathcal{V}_{k}$ to a function in $\mathcal{V}$. Let $\chi$ be a $C_{0}^{\infty}(\mathbb{R})$ cut-off function satisfying

$$
\begin{aligned}
\chi(s) & =1, \quad|s| \leq k / 2, \\
\chi(s) & =0, \quad|s| \geq(k+1) / 2, \\
\chi^{\prime}, \chi^{\prime \prime} & \leq C_{0}
\end{aligned}
$$

A. Pankov and J. Plugger considered in [2] the cut-off operator

$$
\left(P_{k} v\right)(x, y)=\partial_{x}\left[\chi(x) \partial_{x, k}^{-1} v(x, y)\right], \quad \partial_{x, k}^{-1} v(x, y)=\int_{-k / 2}^{x} v(r, y) d r .
$$

Using this operator, it is possible to establish an extension operator $E_{k}$ from $X_{k}$ to $x$. 
LEMMA 2.2.

1. Let $S_{k}$ be the operator defined on $\mathcal{V}_{k}$ by

$$
S_{k} u=\partial_{x}^{-1}\left[P_{k}\left(\partial_{x} u\right)\right], \quad \partial_{x}^{-1} w(x, y)=\int_{-\infty}^{x} w(r, y) d r
$$

Then $S_{k}$ is a uniformly bounded (with respect $k$ ) linear operator from $\mathcal{V}_{k}$ into $\mathcal{V}$, and $\left.S_{k}\right|_{Q_{k}} u=u$.

2. Let $F_{k}$ be the operator defined on $H_{p e r}^{1}\left(\mathbb{R}^{2}\right)$ by $F_{k}(u)=\chi(x) u(x, y)$. Then $F_{k}$ is a uniformly bounded (with respect $k$ ) linear operator from $H_{k}^{1}\left(\mathbb{R}^{2}\right)$ into $H^{1}\left(\mathbb{R}^{2}\right)$, and $\left.F_{k}\right|_{Q_{k}} u=u$.

3. $E_{k}=\left(F_{k}, S_{k}\right)$ defines a uniformly bounded (with respect $k$ ) linear operator from $X_{k}$ into $X$ such that $\left.E_{k}\right|_{Q_{k}} U=U$.

The first part was proved by J. Quintero in [4]. The second one is straightforward. From this result and Lemma (2.1) we obtain the corresponding embedding in $\mathcal{V}_{k}$.

Lemma 2.3. (J. Quintero [4]) For $q \geq 2$, we have that

1. The embedding $\mathcal{V}_{k} \hookrightarrow \mathcal{M}^{q}\left(Q_{k}\right)$ is continuous with the embedding constants being uniformly bounded with respect to $k$ and the embedding $\mathcal{V}_{k} \hookrightarrow \mathcal{M}_{l o c}^{q}\left(Q_{k}\right)$ is compact.

2. The embedding $X_{k} \hookrightarrow L^{q}\left(Q_{k}\right) \times \mathcal{N}^{q}\left(Q_{k}\right)$ is continuous with the constants being uniformly bounded with respect to $k$ and the embedding $X_{k} \hookrightarrow L_{l o c}^{q}\left(Q_{k}\right) \times$ $\mathcal{M}_{\text {loc }}^{(q)}\left(Q_{k}\right)$ is compact.

2.2. Existence of $x$-periodic travelling waves. The result is a consequence of a variational approach applied to a suitable minimax, since solutions $(u, v)$ of $(2.4)$ are critical points of the functional $J_{c, k}$ given by

$$
J_{c, k}=\mathcal{J}_{k}(u, v)+G_{1, k}(u, v)+G_{2, k}(u, v)=\Sigma_{k}(u, v)+G_{2, k}(u, v),
$$

where the functionals $\mathcal{J}_{k}, G_{1, k}$, and $G_{2, k}$ are defined on the space $X_{k}$ by

$$
\begin{aligned}
\mathcal{J}_{k}(u, v) & =\int_{Q_{k}}\left(u^{2}+\left(\sigma-\frac{1}{2}\right)|\nabla u|^{2}+|\nabla v|^{2}+\frac{1}{6}|\Delta v|^{2}\right) d y d x \\
G_{1, k}(u, v) & =-2 c \int_{Q_{k}} u v_{x} d y d x \\
G_{2, k}(u, v) & =\frac{2}{p+1} \int_{Q_{k}} u\left(v_{x}^{p+1}+v_{y}^{p+1}\right) d y d x .
\end{aligned}
$$

First we have that $\mathcal{J}_{k}, G_{1, c}, G_{2, k}, J_{c, k} \in C^{2}\left(X_{k}, \mathbb{R}\right)$ and its derivatives in $(u, v)$ in the direction of $(U, V)$ are given by

$$
\begin{aligned}
\left\langle J_{k}^{\prime}(u, v),(U, V)\right\rangle & =2 \int_{Q_{k}}\left(u U+\left(\sigma-\frac{1}{2}\right) \nabla u \cdot \nabla U+\nabla v \cdot \nabla V+\frac{1}{6} \Delta v \Delta V\right) d x d y \\
\left\langle G_{1, c}^{\prime}(u, v),(U, V)\right\rangle & =-2 c \int_{Q_{k}}\left(u V_{x}+v_{x} U\right) d x d y \\
\left\langle G_{2}^{\prime}(u, v),(U, V)\right\rangle & =\frac{2}{p+1} \int_{Q_{k}}\left[\left(v_{x}^{p+1}+v_{y}^{p+1}\right) U+(p+1) u\left(v_{x}^{p} V_{x}+v_{y}^{p} V_{y}\right)\right] d x d y .
\end{aligned}
$$


As a consequence of this we conclude that

$$
J_{k}^{\prime}(u, v)=2\left(\begin{array}{c}
u-\left(\sigma-\frac{1}{2}\right) \Delta u+\frac{1}{p+1}\left(v_{x}^{p+1}+v_{y}^{p+1}\right)-c v_{x} \\
-\Delta v+\frac{1}{6} \Delta^{2} v-\nabla \cdot\left(u\left(v_{x}^{p}, v_{y}^{p}\right)\right)+c u_{x}
\end{array}\right),
$$

meaning that critical points of the functional $J_{c, k}$ satisfy the travelling wave equation (2.4). Hereafter, we will say that weak solutions for (2.4) are critical points of the functional $J_{c, k}$. In particular, we have that

$$
\begin{aligned}
\left\langle J_{c, k}^{\prime}(u, v),(u, v)\right\rangle & =2 \mathcal{J}_{k}(u, v)+2 G_{1, k}(u, v)+(p+2) G_{2, k}(u, v) \\
& =2 J_{c, k}(u, v)+p G_{2, k}(u, v) .
\end{aligned}
$$

Thus on any critical point $(u, v)$, we have that

$$
\begin{aligned}
& J_{c, k}(u, v)=\left(\frac{p}{p+2}\right) \Sigma_{k}(u, v), \\
& J_{c, k}(u, v)=-\left(\frac{p}{2}\right) G_{2, k}(u, v), \\
& \Sigma_{k}(u, v)=-\left(\frac{p+2}{2}\right) G_{2, k}(u, v) .
\end{aligned}
$$

One can see easily that the functionales $G_{1, k}$ and $G_{2, k}$ are well-defined on $X_{k}$. Note that $u, v_{x}, v_{y} \in H^{1}\left(Q_{k}\right) \subset L^{q}\left(Q_{k}\right)$ for all $q \geq 2$, therefore by applying Young's inequality to the second members of $G_{1, k}(u, v)$ and $G_{2, k}(u, v)$ we obtain that

$$
\left|G_{1, k}(u, v)\right| \leq|c| \int_{Q_{k}}\left\{u^{2}+\left|v_{x}\right|^{2}\right\} d x d y \leq|c|\|(u, v)\|_{x_{k}}^{2}
$$

and,

$$
\left|G_{2, k}(u, v)\right| \leq C_{1}(p)\|(u, v)\|_{x_{k}}^{p+2} .
$$

Now, for $\sigma>1 / 2$ and $0<|c|<1$ we have from inequality (2.9) that there are some positive constants $C_{1}(\sigma, c)<C_{2}(\sigma, c)$ such that

$$
\begin{aligned}
\Sigma_{k}(u, v) & =\int_{Q_{k}}\left(u^{2}+\left(\sigma-\frac{1}{2}\right)|\nabla u|^{2}+|\nabla v|^{2}+\frac{1}{6}|\Delta v|^{2}\right) d y d x+G_{1, k}(u, v) \\
& \leq \int_{Q_{k}}\left\{(1+|c|) u^{2}+\left(\sigma-\frac{1}{2}\right)|\nabla u|^{2}+(1+|c|)\left|v_{x}\right|^{2}+\left|v_{y}\right|^{2}+\frac{1}{6}|\Delta v|^{2}\right\} d x d y \\
& \leq C_{2}(\sigma, c)\|(u, v)\|_{x_{k}}^{2}
\end{aligned}
$$

In a similar fashion, we have that

$$
\begin{aligned}
\Sigma_{k}(u, v) & =\int_{Q_{k}}\left(u^{2}+\left(\sigma-\frac{1}{2}\right)|\nabla u|^{2}+|\nabla v|^{2}+\frac{1}{6}|\Delta v|^{2}\right) d y d x+G_{1, k}(u, v) \\
& \geq \int_{Q_{k}}\left\{(1-|c|) u^{2}+\left(\sigma-\frac{1}{2}\right)|\nabla u|^{2}+(1-|c|)\left|v_{x}\right|^{2}+\left|v_{y}\right|^{2}+\frac{1}{6}|\Delta v|^{2}\right\} d x d y \\
& \geq C_{1}(\sigma, c)\|(u, v)\|_{x_{k}}^{2}
\end{aligned}
$$

In other words, we have shown for $\sigma>1 / 2$ and $0<|c|<1$ that

$$
C_{1}(\sigma, c)\|(u, v)\|_{X_{k}}^{2} \leq \Sigma_{k}(u, v) \leq C_{2}(\sigma, c)\|(u, v)\|_{\mathcal{X}_{k}}^{2} .
$$


Our approach to show the existence of a non trivial critical point for $J_{c, k}$ is to use the Mountain Pass Lemma without the Palais-Smale condition (see Willem ( [16], Theorem 2.8), Ambrosetti et al. [17]) to build a Palais-Smale sequence for $J_{c, k}$ for a minimax value and use a local embedding result to obtain a critical point for $J_{c, k}$ as a weak limit of such Palais-Smale sequence.

TheOrem 2.4. Let $X$ be a Hilbert space, $\varphi \in C^{1}(X, \mathbb{R}), e \in X$ and $r>0$ such that $\|e\|_{X}>r$ and

$$
b=\inf _{\|u\|_{X}=r} \varphi(u)>\varphi(0) \geq \varphi(e) .
$$

Then, given $n \in \mathbb{N}$, there is $u_{n} \in X$ such that

$$
\varphi\left(u_{n}\right) \rightarrow d, \text { and } \varphi^{\prime}\left(u_{n}\right) \rightarrow 0 \text { in } X^{\prime},
$$

where

$$
d=\inf _{\gamma \in \Gamma} \max _{t \in[0,1]} \varphi(\gamma(t)), \quad \text { and } \Gamma=\{\gamma \in C([0,1], X): \gamma(0)=0, \quad \gamma(1)=e\} .
$$

Before we go further, we establish an important result for our analysis, which is related the characterization of "vanishing" sequences in $X_{k}$. Define the density $\varrho_{1}$ on $x_{k}$ as

$$
\varrho_{1}(u, v)=u^{2}+|\nabla v|^{2}
$$

and for $r>0$ and $\zeta \in \mathbb{R}$ define the rectangle

$$
R_{r, k}(\zeta)=\left[-\frac{k}{2}, \frac{k}{2}\right] \times[\zeta-r, \zeta+r]
$$

TheOREM 2.5. Let $q \geq 2$. If $\left(u_{n}, v_{n}\right)_{n}$ is a bounded sequence in $X_{k}$ and there is a positive constant $r>0$ such that

$$
\lim _{n \rightarrow \infty} \sup _{\zeta \in \mathbb{R}}\left(\int_{R_{r, k}(\zeta)} \varrho_{1}\left(u_{n}, v_{n}\right) d y d x\right)=0
$$

then we have that

$$
\lim _{n \rightarrow \infty}\left\|v_{n}\right\|_{\mathcal{M}^{(q)}\left(Q_{k}\right)}=\lim _{n \rightarrow \infty}\left\|u_{n}\right\|_{L^{q}\left(Q_{k}\right)}=0
$$

Proof. First suppose that $\left(w_{n}\right)_{n}$ is a bounded sequence in $H^{1}\left(Q_{k}\right)$ and assume there is a positive constant $r>0$ such that

$$
\lim _{n \rightarrow \infty} \sup _{\zeta \in \mathbb{R}} \int_{R_{r, k}(\zeta)} w_{n}^{2} d y d x=0 .
$$

We will see that $\lim _{n \rightarrow \infty}\left\|w_{n}\right\|_{L^{q}\left(Q_{k}\right)}=0$. In fact, let $\left(w_{n}\right)_{n}$ be a bounded sequence in $H^{1}\left(Q_{k}\right)$ satisfying the limit $(2.14)$. Then, from the Hölder inequality and the embedding Lemma (2.1) we have for $q \geq 2$ that

$$
\left\|w_{n}\right\|_{L^{q}\left(R_{r, k}(\zeta)\right)}^{q} \leq\left\|w_{n}\right\|_{L^{2}\left(R_{r, k}(\zeta)\right)}\left\|w_{n}\right\|_{L^{2(q-1)}\left(R_{r, k}(\zeta)\right)}^{q-1} \leq\left\|w_{n}\right\|_{\left.L^{2}\left(R_{r, k}(\zeta)\right)\right)}\left\|w_{n}\right\|_{H^{1}\left(Q_{k}\right)}^{q-1} .
$$


Covering $Q_{k}$ by a countable number of rectangles such that every point in $Q_{k}$ is contained in at most 3 rectangles $R_{r, k}(\zeta)$, we obtain that

$$
\left\|w_{n}\right\|_{L^{q}\left(Q_{k}\right)}^{q} \leq 3 \sup _{\zeta \in \mathbb{R}}\left\|w_{n}\right\|_{L^{2}\left(R_{r, k}(\zeta)\right)}\left\|w_{n}\right\|_{H^{1}\left(Q_{k}\right)}^{q-1} .
$$

We conclude using the hypothesis and that $\left(w_{n}\right)_{n}$ is a bounded sequence in $H^{1}\left(Q_{k}\right)$ that

$$
\lim _{n \rightarrow \infty}\left\|w_{n}\right\|_{L^{q}\left(Q_{k}\right)}=0 .
$$

Now suppose that $\left(u_{n}, v_{n}\right) \in X_{k}$ satisfies (2.13), then $u_{n}, \partial_{x} v_{n}, \partial_{y} v_{n} \in H^{1}\left(Q_{k}\right)$. If $w_{n}$ is defined as either $u_{n}, \partial_{x} v_{n}$, or $\partial_{y} v_{n}$, we see that $w_{n}$ satisfies in each case the condition (2.14). By previous observation, we conclude for $q \geq 2$ that $\lim _{n}\left\|w_{n}\right\|_{L^{q}\left(Q_{k}\right)}=0$. In other words, we have for $q \geq 2$ that,

$$
\lim _{n \rightarrow \infty}\left\|u_{n}\right\|_{L^{q}\left(Q_{k}\right)}=\lim _{n \rightarrow \infty}\left\|v_{n}\right\|_{\mathcal{M}^{(q)}\left(Q_{k}\right)}=0 .
$$

Now, we want to verify the Mountain Pass Theorem hypotheses given in Theorem (2.4) and to build a Palais-Smale sequence for $J_{c, k}$.

Lemma 2.6. Let $0<|c|<1$ and $\sigma>1 / 2$,

1. there exists $\rho>0$ small enough such that $b(c):=\inf _{\|z\|_{x_{k}}=\rho} J_{c, k}(z)>0$.

2. there is $e \in X_{k}$ with $\|e\|_{X_{k}} \geq \rho$ such that $J_{c, k}(e) \leq 0$.

3. If $d(c)$ is defined as

$$
d(c)=\inf _{\gamma \in \Gamma} \max _{t \in[0,1]} J_{c, k}(\gamma(t)), \quad \Gamma=\left\{\gamma \in C\left([0,1], x_{k}\right) \mid \gamma(0)=0, \gamma(1)=e\right\},
$$

then $d(c) \geq b(c)$ and there is a sequence $\left(U_{n}\right)_{n} \in X_{k}$ such that

$$
J_{c, k}\left(U_{n}\right) \rightarrow d, \quad J_{c, k}^{\prime}\left(U_{n}\right) \rightarrow 0 \quad \text { in } \quad X_{k}^{\prime}
$$

Proof. From inequalities (2.9), (2.10), and (2.11), we have for any $(u, v) \in X_{k}$ that

$$
\begin{aligned}
J_{c, k}(u, v) & \geq C_{1}(\sigma, c)\|(u, v)\|_{X_{k}}^{2}-C_{1}(p)\|(u, v)\|_{X_{k}}^{p+2} \\
& \geq\left(C_{1}(\sigma, c)-C_{1}(p)\|(u, v)\|_{X_{k}}^{p}\right)\|(u, v)\|_{X_{k}}^{2}
\end{aligned}
$$

Then for $\rho>0$ small enough such that

$$
C_{1}(\sigma, c)-C_{1}(p) \rho^{p}>0
$$

we conclude for $\|(u, v)\|_{x_{k}}=\rho$ that

$$
J_{c, k}(u, v) \geq\left(C_{1}(\sigma, c)-C_{1}(p) \rho^{p}\right) \rho^{2}:=\delta>0 .
$$

In particular, we also have that

$$
b(c)=\inf _{\|z\|_{x_{k}}=\rho} J_{c, k}(z) \geq \delta>0 .
$$


Now, it is not hard to prove that there exist $u_{0}, v_{0} \in C_{0}^{\infty}\left(Q_{k}\right)$ such that $G_{2, k}\left(u_{0}, v_{0}\right)<$ 0 . Then for any $t \in \mathbb{R}$ we have that

$$
\begin{aligned}
J_{c, k}\left(t u_{0}, t v_{0}\right) & =t^{2} \mathcal{J}_{k}\left(u_{0}, v_{0}\right)+t^{2} G_{1, c}\left(u_{0}, v_{0}\right)+t^{p+2} G_{2, k}\left(u_{0}, v_{0}\right) \\
& =t^{2}\left[\mathcal{J}_{k}\left(u_{0}, v_{0}\right)+G_{1, c}\left(u_{0}, v_{0}\right)+t^{p} G_{2, k}\left(u_{0}, v_{0}\right)\right] .
\end{aligned}
$$

As a consequence of this, we have that

$$
\lim _{t \rightarrow \infty} J_{c, k}\left(t u_{0}, t v_{0}\right)=-\infty
$$

and so, there is $t_{0}>0$ such that $e=t_{0}\left(u_{0}, v_{0}\right) \in X_{k}$ satisfies that $t_{0}\left\|\left(u_{0}, v_{0}\right)\right\| x_{k}=$ $\|e\|_{x_{k}}>\rho$ and that $J(e) \leq J(0)=0$. The third part follows by applying Theorem $(2.4)$.

TheOrem 2.7. For $0<|c|<1, \sigma>1 / 2$ and $k>0$, problem (2.4) has a nontrivial solution in $X_{k}$.

Proof. Let $\left(u_{n}, v_{n}\right) \in X_{k}$ be the sequence given by previous Lemma. First note that $d(c) \geq b(c) \geq \delta$ from (2.16). Using the definition of $J_{c, k}$ and (2.5) we have that

$$
\Sigma_{k}\left(u_{n}, v_{n}\right)=\left(\frac{p+2}{p}\right) J_{c, k}\left(u_{n}, v_{n}\right)-\frac{1}{p}\left\langle J_{c, k}^{\prime}\left(u_{n}, v_{n}\right),\left(u_{n}, v_{n}\right)\right\rangle .
$$

But from (2.11) we conclude for $n$ large enough that

$$
C_{1}(\sigma, c)\left\|\left(u_{n}, v_{n}\right)\right\|_{x_{k}}^{2} \leq \Sigma_{k}\left(u_{n}, v_{n}\right) \leq\left(\frac{p+2}{p}\right)(d(c)+1)+\left\|\left(u_{n}, v_{n}\right)\right\| x_{k} .
$$

Then we have shown that $\left(u_{n}, v_{n}\right)_{n}$ is a bounded sequence in $X_{k}$. We claim that

$$
\delta^{*}=\varlimsup_{n \rightarrow \infty} \sup _{\zeta \in \mathbb{R}} \int_{R_{1, k}(\zeta)} \varrho_{1}\left(u_{n}, v_{n}\right) d x d y>0 .
$$

If we suppose that

$$
\varlimsup_{n \rightarrow \infty} \sup _{\zeta \in \mathbb{R}} \int_{R_{1, k}(\zeta)} \varrho_{1}\left(u_{n}, v_{n}\right) d x d y=0 .
$$

Then from Theorem (2.5) we conclude for $q \geq 2$ that

$$
\lim _{n \rightarrow \infty}\left\|u_{n}\right\|_{L^{q}\left(Q_{k}\right)}=0, \quad \lim _{n \rightarrow \infty}\left\|v_{n}\right\|_{\mathcal{M}^{(q)}\left(Q_{k}\right)}=0 .
$$

Now, we have from (2.16) and (2.10) that

$$
\begin{aligned}
0<\delta \leq d(c) & =J_{c, k}\left(u_{n}, v_{n}\right)-\frac{1}{2}\left\langle J_{c, k}^{\prime}\left(u_{n}, v_{n}\right),\left(u_{n}, v_{n}\right)\right\rangle+o(1) \\
& =\frac{p}{2} G_{2, k}\left(u_{n}, v_{n}\right)+o(1) \\
& \leq C(p)\left[\left\|u_{n}\right\|_{L^{p+2}\left(Q_{k}\right)}^{p+2}+\left\|v_{n}\right\|_{\mathcal{M}^{(p+2)}\left(Q_{k}\right)}^{p+2}\right]+o(1) \\
& \leq o(1)
\end{aligned}
$$


but this is a contradiction. Thus, there is a subsequence of $\left(u_{n}, v_{n}\right)_{n}$, denoted the same, and a sequence $\zeta_{n} \in \mathbb{R}$

$$
\int_{R_{1, k}\left(\zeta_{n}\right)} \varrho_{1}\left(u_{n}, v_{n}\right) d x d y \geq \frac{\delta^{*}}{2}
$$

Now we define the sequence

$$
\left(\tilde{u}_{n}, \tilde{v}_{n}\right)\left(\zeta_{1}, \zeta_{2}\right)=\left(u_{n}, v_{n}\right)\left(\zeta_{1}, \zeta_{2}+\zeta_{n}\right)
$$

For this sequence we also have that

$$
\left\|\left(\tilde{u}_{n}, \tilde{v}_{n}\right)\right\|_{x_{k}}=\left\|\left(u_{n}, v_{n}\right)\right\|_{x_{k}}, \quad J_{c, k}\left(\tilde{u}_{n}, \tilde{v}_{n}\right) \rightarrow d, \quad J_{c, k}^{\prime}\left(\tilde{u}_{n}, \tilde{v}_{n}\right) \rightarrow 0 \text { in } x_{k}^{\prime} .
$$

So, we also have the existence of a subsequence of $\left(\tilde{u}_{n}, \tilde{v}_{n}\right)_{n}$, denoted the same, and some $(u, v) \in X_{k}$, we have that

$$
\left(\tilde{u}_{n}, \tilde{v}_{n}\right) \rightarrow(u, v), \quad \text { as } n \rightarrow \infty \quad\left(\text { weakly in } X_{k}\right) .
$$

From Lemma (2.3), we have the embedding $X_{k} \hookrightarrow L_{l o c}^{q}\left(Q_{k}\right) \times \mathcal{M}_{l o c}^{(q)}\left(Q_{k}\right)$ is locally compact for $q \geq 2$. Then the weakly convergent sequence $\left(\tilde{u}_{n}, \tilde{v}_{n}\right)_{n}$ in $X_{k}$ has a subsequence (denoted the same) than converges strongly to $(u, v)$ in $L_{l o c}^{q}\left(Q_{k}\right) \times \mathcal{M}_{l o c}^{(q)}\left(Q_{k}\right)$. On the other hand, for $q=2$ and using inequality (2.11), we have that

$$
C_{2}(\sigma, c)\|(u, v)\|_{\mathfrak{X}_{k}}^{2} \geq \int_{R_{1, k}(0)} \varrho_{1}(u, v) d x d y=\lim _{n \rightarrow \infty} \int_{R_{1, k}(0)} \varrho_{1}\left(\tilde{u}_{n}, \tilde{v}_{n}\right) d x d y \geq \frac{\delta^{*}}{2}
$$

So, form previous fact we conclude that $(u, v) \neq 0$. Moreover, if $Z=(U, V) \in\left(C_{0}^{\infty}\right)^{2}$, then for $K=\operatorname{supp} Z$ we have that

$$
\begin{aligned}
\left\langle\mathcal{J}_{k}^{\prime}(u, v), Z\right\rangle & =2 \int_{K}\left(u U+\left(\sigma-\frac{1}{2}\right) \nabla u \cdot \nabla U+\nabla v \cdot \nabla V+\frac{1}{6} \Delta v \Delta V\right) d x d y \\
& =2 \lim _{n \rightarrow \infty} \int_{K}\left(\tilde{u}_{n} U+\left(\sigma-\frac{1}{2}\right) \nabla \tilde{u}_{n} \cdot \nabla U+\nabla \tilde{v}_{n} \cdot \nabla V+\frac{1}{6} \Delta \tilde{v}_{n} \Delta V\right) d x d y \\
& =\lim _{n \rightarrow \infty}\left\langle\mathcal{J}_{k}^{\prime}\left(\tilde{u}_{n}, \tilde{v}_{n}\right), Z\right\rangle \\
\left\langle G_{1, k}^{\prime}(u, v), Z\right\rangle & =-2 c \int_{K}\left(u V_{x}+v_{x} U\right) d x d y=-2 c \lim _{n \rightarrow \infty} \int_{Q_{k}}\left(\tilde{u}_{n} V_{x}+\left(\tilde{v}_{n}\right)_{x} U\right) d x d y \\
& =\lim _{n \rightarrow \infty}\left\langle G_{1, k}^{\prime}\left(\tilde{u}_{n}, \tilde{v}_{n}\right), Z\right\rangle .
\end{aligned}
$$

Now noting that the sequences $\left(\left(\partial_{i} \tilde{v}_{n}\right)^{p+1}\right)_{n}$ and $\left(\tilde{u}_{n}\left(\partial_{i} \tilde{v}_{n}\right)^{p}\right)_{n}$ are bounded in $L^{2}\left(Q_{k}\right)$, then (taking a subsequence, if necessary), we have that

$$
\left(\partial_{i} \tilde{v}_{n}\right)^{p+1} \rightarrow\left(\partial_{i} v\right)^{p+1} \text { and } \tilde{u}_{n}\left(\partial_{i} \tilde{v}_{n}\right)^{p} \rightarrow u\left(\partial_{i} v\right)^{p} \text { in } L^{2}\left(Q_{k}\right)
$$

As a consequence of this, we have that

$$
\begin{aligned}
\int_{K}\left(\left(\tilde{v}_{n}\right)_{x}^{p+1}+\left(\tilde{v}_{n}\right)_{y}^{p+1}\right) U d x d y & \rightarrow \int_{K}\left(v_{x}^{p+1}+v_{y}^{p+1}\right) U d x d y \\
\int_{K} \tilde{u}_{n}\left(\left(\tilde{v}_{n}\right)_{x}^{p} V_{x}+\left(\tilde{v}_{n}\right)_{y}^{p} V_{y}\right) d x d y & \rightarrow \int_{K} u\left(v_{x}^{p} V_{x}+v_{y}^{p} V_{y}\right) d x d y
\end{aligned}
$$


In other words, we have shown that

$$
\left\langle G_{2, k}^{\prime}(u, v),(U, V)\right\rangle=\lim _{n \rightarrow \infty}\left\langle G_{2, k}^{\prime}\left(\tilde{u}_{n}, \tilde{v}_{n}\right), Z\right\rangle,
$$

and also that

$$
\left\langle J_{c, k}^{\prime}(u, v), Z\right\rangle=\lim _{n \rightarrow \infty}\left\langle J_{c, k}^{\prime}\left(\tilde{u}_{n}, \tilde{v}_{n}\right), Z\right\rangle=0 .
$$

Let $Z \in X_{k}$. By density, there is $Z_{k} \in\left(C_{0}^{\infty}\right)^{2}$ such that $Z_{k} \rightarrow Z$ in $X_{k}$. Then

$$
\begin{aligned}
\left|\left\langle J_{c, k}^{\prime}(u, v), Z\right\rangle\right| & \leq\left|\left\langle J_{c, k}^{\prime}(u, v), Z-Z_{k}\right\rangle\right|+\left|\left\langle J_{c, k}^{\prime}(u, v), Z_{k}\right\rangle\right| \\
& \leq\left\|J_{c, k}^{\prime}(u, v)\right\|_{x_{k}{ }^{\prime}}\left\|Z-Z_{k}\right\| x_{k}+\left|\left\langle J_{c, k}^{\prime}(u, v), Z_{k}\right\rangle\right| .
\end{aligned}
$$

Thus we have already established that $J_{c, k}^{\prime}(u, v)=0$. In other words, $(u, v)$ is a nontrivial solution for problem (2.4).

Now, we present a variational characterization of the critical value $d(c)$. To do so, we want to characterize $x$-periodic travelling waves solutions of (2.4) through a minimization problem. We must note that critical points $U \in X_{k}$ for $J_{c, k}$ satisfies that $J_{c, k}^{\prime}(U)(V)=0$ for any $V \in X_{k}$. So, we need to look for critical point in the Nehari manifold defined by

$$
\mathcal{N}_{c, k}=\left\{U \in X_{k}: \Lambda_{k}(U)=0\right\},
$$

where

$$
\Lambda_{k}(U)=J_{c, k}^{\prime}(U)(U)=2 \Sigma_{k}(U)+(p+2) G_{2, k}(U) .
$$

Our interest for $k>0$ is to establish the existence of $U_{0} \in \mathcal{N}_{c, k}$ such that

$$
J_{c, k}\left(U_{0}\right)=\inf \left\{J_{c, k}(U): U \in \mathcal{N}_{c, k}\right\}:=\mathcal{J}_{k}(c) .
$$

One of the main ingredients is the variational characterization of $\mathcal{J}_{k}(c)$ and $d(c)$ given by the following result.

LEMma 2.8. For $0<|c|<1$ and $\sigma>\frac{1}{2}$, we have that

$$
d(c)=\mathcal{J}_{k}(c)=\left(\frac{2^{\frac{2}{p}} p}{(p+2)^{\frac{p+2}{p}}}\right) \Upsilon(c)^{\frac{p+2}{p}}
$$

where $\mathcal{J}_{k}(c)$ and $\Upsilon_{k}(c)$ are defined as

$$
\begin{aligned}
& \mathcal{J}_{k}(c)=\inf \left\{\left(\frac{p}{p+2}\right) \Sigma_{k}(U): \Lambda_{k}(U)=0, \quad U \neq 0\right\} \\
& \Upsilon_{k}(c)=\inf \left\{\Sigma_{k}(U): G_{2}(U)=1, \quad U \in X_{k}\right\} .
\end{aligned}
$$

Moreover, we have that

$$
\mathcal{J}_{k}(c)=\inf \left\{\left(\frac{p}{p+2}\right) \Sigma_{k}(U): \Lambda_{k}(U) \leq 0, \quad U \neq 0\right\} .
$$

Proof. We first note that from Theorem (2.7), we have that $d(c)$ is a critical value and that there is in $X_{k}$ a non trivial critical point for $J_{c, k}$, meaning that $\left\{U \in X_{k}: \Lambda_{k}(U)=0\right\}$ is not an empty set. Moreover, we also have that

$$
\partial_{k}(c)=\inf \left\{J_{c, k}(U): \Lambda_{k}(U)=0, \quad U \neq 0\right\} \leq d(c) .
$$


Now, from (2.11) we show that $\mathcal{J}_{k}$ exists and is positive. Now we will establish that

$$
\mathcal{J}_{k}(c)=\left(\frac{2^{\frac{2}{p}} p}{(p+2)^{\frac{p+2}{p}}}\right) \Upsilon_{k}(c)^{\frac{p+2}{p}} .
$$

Let $U \in X_{k} \backslash\{0\}$ be such that $\Lambda_{k}(U)=0$. Then we have that $G_{2, k}(U)<0$ and

$$
\Sigma_{k}(U)=-\left(\frac{p+2}{2}\right) G_{2, k}(U)=\left(\frac{p+2}{2}\right)\left|G_{2, k}(U)\right|=\left(\frac{p+2}{p}\right) J_{c, k}(U) .
$$

Now we consider $W=\left(\frac{1}{G_{2, k}(U)}\right)^{\frac{1}{p+2}} U$. Thus, by definition we have that

$$
\begin{aligned}
\Upsilon_{k}(c) & \leq \Sigma_{k}(W)=\left(\frac{1}{\left|G_{2, k}(U)\right|}\right)^{\frac{2}{p+2}} \Sigma_{k}(U) \\
& \leq\left(\frac{p+2}{2}\right)^{\frac{2}{p+2}}\left(\Sigma_{k}(U)\right)^{\frac{p}{p+2}} \\
& \leq\left(\frac{p+2}{2}\right)^{\frac{2}{p+2}}\left(\frac{p+2}{p}\right)^{\frac{p}{p+2}}\left(\left(\frac{p}{p+2}\right) \Sigma_{k}(U)\right)^{\frac{p}{p+2}},
\end{aligned}
$$

meaning that

$$
\left(\frac{2^{\frac{2}{p}} p}{(p+2)^{\frac{p+2}{p}}}\right) \Upsilon_{k}(c)^{\frac{p+2}{p}} \leq \mathcal{J}_{k}(c) .
$$

Now, suppose that $U \neq 0$ is such that $G_{2, k}(U)=1$. Now, for $\alpha \neq 0$ we have that

$$
\Lambda_{k}(\alpha U)=\alpha^{2}\left(2 \Sigma_{k}(U)+(p+2) \alpha^{p} G_{2, k}(U)\right) .
$$

So, taking $\alpha$ such $2 \Sigma_{k}(U)+(p+2) \alpha^{p} G_{2, k}(U)=0$, we have that $\Lambda_{k}(\alpha U)=0$. In this case, we have that

$$
\alpha^{2}=\left[\left(\frac{2}{p+2}\right) \Sigma_{k}(U)\right]^{\frac{2}{p}}
$$

Moreover, we also have that

$$
\begin{aligned}
\mathcal{J}_{k}(c) & \leq\left(\frac{p}{p+2}\right) \Sigma_{k}(\alpha U)=\alpha^{2}\left(\frac{p}{p+2}\right) \Sigma_{k}(U) \\
& \leq \alpha^{2}\left(\frac{p}{p+2}\right) \Sigma_{k}(U) \\
& \leq\left(\frac{2^{\frac{2}{p}} p}{(p+2)^{\frac{p+2}{p}}}\right)\left(\Sigma_{k}(U)\right)^{\frac{p+2}{p}} .
\end{aligned}
$$

In other words, we have shown that

$$
\partial_{k}(c) \leq\left(\frac{2^{\frac{2}{p}} p}{(p+2)^{\frac{p+2}{p}}}\right) \Upsilon_{k}(c)^{\frac{p+2}{p}}
$$


and so

$$
\left(\frac{2^{\frac{2}{p}} p}{(p+2)^{\frac{p+2}{p}}}\right) \Upsilon_{k}(c)^{\frac{p+2}{p}}=\mathcal{J}_{k}(c) \leq d(c) .
$$

Finally, we will see that $d(c) \leq \mathcal{J}_{k}(c)$. From Lemma (2.6), we have the existence of $e \in X_{k}$ such that $J_{c}(e)<0$. Moreover, $G_{2, k}(e)<0$. Now, we can choose $t_{0}>0$ such that $G_{2, k}\left(-t_{0} e\right)=1$. In fact, this follows by noting that $p=\frac{p_{1}}{p_{2}}$ with $p_{1}$ and $p_{2}$ being odd positive integers, and that $G_{2, k}\left(-t_{0} e\right)=-t_{0}^{p+2} G_{2, k}(e)=1$, which means that $t_{0}=\left(-G_{2, k}(e)\right)^{-\frac{1}{p+2}}>0$. Now, we define a continuous function $\gamma_{0}:[0,1] \rightarrow X_{k}$ as $\gamma_{0}(t)=-\frac{t}{t_{0}} U$, where $U=-t_{0} e$. Note that $\gamma_{0}(0)=0$ and $\gamma_{0}(1)=e$. By the definition of $d(c)$, we conclude that

$$
d(c) \leq \max _{t \in[0,1]} J_{c, k}\left(\gamma_{0}(t)\right) \leq \max _{s \in[0, \infty)} J_{c, k}(-s U)=\max _{s \in[0, \infty)} g(s),
$$

where $g(s)=s^{2} \Sigma_{k}(u, v)-s^{p+2}$. A simple computation shows that $g$ has a unique critical point $s_{0}$ satisfying

$$
s_{0}^{p}=\left(\frac{2}{p+2}\right) \Sigma_{k}(U)
$$

Moreover,

$$
\max _{s \in[0, \infty)} g(s)=g\left(s_{0}\right)=s_{0}^{2}\left(\Sigma_{k}(U)-s_{0}^{p}\right)=\left(\frac{2^{\frac{2}{p}} p}{(p+2)^{\frac{p+2}{p}}}\right)\left(\Sigma_{k}(U)\right)^{\frac{p+2}{p}} .
$$

Then we have shown that

$$
d(c) \leq\left(\frac{2^{\frac{2}{p}} p}{(p+2)^{\frac{p+2}{p}}}\right)\left(\Sigma_{k}(U)\right)^{\frac{p+2}{p}} .
$$

On the other hand, it is not difficult to prove that $d(c)$ is independent of $e$. Let $U_{1} \in X_{k}$ be such that $G_{2, k}\left(U_{1}\right)=1$. Then we know that

$$
J_{c, k}\left(-t U_{1}\right)=t^{2}\left[\Sigma_{k}\left(U_{1}\right)-t^{p} G_{2, k}\left(U_{1}\right)\right] \rightarrow-\infty, \text { as } t \rightarrow \infty .
$$

This guarantees the existence of $U_{1}=-t_{1} e_{1} \in X_{k}$ with $e_{1}$ satisfying the requirements of Lemma (2.6). Using this fact and (2.19), we conclude that

$$
d(c) \leq\left(\frac{2^{\frac{2}{p}} p}{(p+2)^{\frac{p+2}{p}}}\right)\left(\Sigma_{k}\left(U_{1}\right)\right)^{\frac{p+2}{p}} .
$$

This implies that

$$
d(c) \leq\left(\frac{2^{\frac{2}{p}} p}{(p+2)^{\frac{p+2}{p}}}\right) \Upsilon_{k}(c)^{\frac{p+2}{p}}
$$

and so,

$$
d(c)=\mathcal{J}_{k}(c)=\left(\frac{2^{\frac{2}{p}} p}{(p+2)^{\frac{p+2}{p}}}\right) \Upsilon_{k}(c)^{\frac{p+2}{p}}
$$


We will prove the last part. Let $U \neq 0$ in $X_{k}$ be such that $\Lambda_{k}(U) \leq 0$. Then, we have that $G_{2, k}(U)<0$. Define $\alpha \in[0,1)$ by

$$
\alpha^{p}=-\left(\frac{2}{p+2}\right)\left(\frac{\Sigma_{k}(U)}{G_{2, k}(U)}\right) .
$$

Then a direct computation shows that $\Lambda_{k}(\alpha U)=0$. So, we conclude that

$$
\inf \left\{J_{k}(U): \Lambda_{k}(U)=0\right\} \leq J_{c, k}(\alpha U)=\frac{\alpha^{2} p}{p+2} \Sigma_{k}(U) \leq \frac{p}{p+2} \Sigma_{k}(U) .
$$

Thus we obtain the first inequality,

$$
\inf \left\{J_{k}(U): \Lambda_{k}(U)=0\right\} \leq \inf \left\{\left(\frac{p}{p+2}\right) \Sigma_{k}(U): \Lambda_{k}(U) \leq 0, \quad U \neq 0\right\} .
$$

Now take $U \in X_{k}$ such that $\Lambda_{k}(U)=0$. Then $J_{k}(U)=\left(\frac{p}{p+2}\right) \Sigma_{k}(U)$. As a consequence of this,

$$
\inf \left\{J_{k}(U): \Lambda_{k}(U)=0\right\} \geq \inf \left\{\left(\frac{p}{p+2}\right) \Sigma_{k}(U): \Lambda_{k}(U) \leq 0, \quad U \neq 0\right\} .
$$

Thus we obtain the conclusion.

Now, we will see how a compactness property is used to prove the existence of a minimizer for $J_{k}(c)$. We set the density

$$
\begin{aligned}
\varrho(u, v) & =u^{2}+\left(\sigma-\frac{1}{2}\right)|\nabla u|^{2}+|\nabla v|^{2}+\frac{1}{6}|\Delta v|^{2}-2 c u v_{x}, \\
& =\left(u-c v_{x}\right)^{2}+\left(\sigma-\frac{1}{2}\right)|\nabla u|^{2}+\left(1-c^{2}\right) v_{x}^{2}+v_{y}^{2}+\frac{1}{6}|\Delta v|^{2} .
\end{aligned}
$$

Lemma 2.9. Let $\left\{U_{n}\right\}_{n}$ be a minimizing sequence for $\mathcal{J}_{k}(c)$. If there exists a sequence of points $\zeta_{n} \in \mathbb{R}$ such that for any $\epsilon>0$ there exists $l>0$ such that

$$
\left(\frac{p}{p+2}\right) \int_{R_{l, k}\left(\zeta_{n}\right)} \varrho\left(U_{n}\right) d x d y \geq J_{k}(c)-\epsilon, \quad \text { for all } n \in \mathbb{N}
$$

then, there exists a subsequence of $\left\{U_{n}\right\}_{n}$ (denoted the same) and a minimizer $U_{0, k}$ for $\mathcal{J}_{k}(c)$ such that the translated sequence $\tilde{U}_{n}=U_{n}\left(\cdot+\left(0, \zeta_{n}\right)\right)$ converges strongly to $U_{0, k}$ in $X_{k}$.

Proof. Note that any $\left\{U_{n}\right\}_{n}$ minimizing sequence for $\mathcal{J}_{k}(c)$ is bounded in $X_{k}$. Define the shifted sequence $\tilde{U}_{n}(\zeta)=U_{n}\left(\zeta+\left(0, \zeta_{n}\right)\right)$. Then $\left\{\tilde{U}_{n}\right\}_{n}$ is also a bounded minimizing for $\mathcal{J}_{k}(c)$ in $X_{k}$. Thus there exists $U_{0, k}=\left(u_{k}, v_{k}\right) \in X_{k}$ and a subsequence of $\tilde{U}_{n}$ (denoted the same) that converges to $U_{0, k}$ weakly in $X_{k}$ and strongly in $L_{l o c}^{q}\left(Q_{k}\right) \times \mathcal{M}_{l o c}^{q}\left(Q_{k}\right)$ for $q \geq 2$. We also have that,

$$
\begin{aligned}
\Lambda_{k}\left(U_{n}\right)= & \Lambda_{k}\left(\tilde{U}_{n}\right)=0, \quad J_{c, k}\left(U_{n}\right)=J_{c, k}\left(\tilde{U}_{n}\right) \rightarrow \mathcal{J}_{k}(c), \quad \text { and } \\
& \left(\frac{p}{p+2}\right) \int_{R_{l, k}(0)} \varrho\left(\tilde{U}_{n}\right) d x d y \geq \mathcal{J}_{k}(c)-\epsilon .
\end{aligned}
$$


Now for $w_{n}=\tilde{u}_{n}$ or $w_{n}=\partial_{i} \tilde{v}_{n}$, we can take $l>0$ such that for $n$ sufficiently large

$$
\int_{R_{l, k}(0)}\left|w_{n}\right|^{2} d x d y \geq \int_{Q_{k}}\left|w_{n}\right|^{2} d x d y-\frac{\epsilon}{2}
$$

where $\tilde{U}_{n}=\left(\tilde{u}_{n}, \tilde{v}_{n}\right)$. Since we have that the local embedding $X_{k} \subset L_{l o c}^{q}\left(Q_{k}\right) \times$ $\mathcal{M}_{l o c}^{q}\left(Q_{k}\right)$ is compact for $q \geq 2$, then we have strong convergence of $w_{n}$ to $w_{k}$ in $L^{2}\left(Q_{k}\right)$, where $w_{k}=u_{k}$ or $w_{k}=\partial_{i} v_{k}$. In fact,

$$
\begin{aligned}
\int_{Q_{k}}\left|w_{k}\right|^{2} d x d y & \leq \liminf _{n \rightarrow \infty} \int_{Q_{k}}\left|w_{n}\right|^{2} d x d y \\
& \leq \liminf _{n \rightarrow \infty} \int_{R_{l, k}(0)}\left|w_{n}\right|^{2} d x d y+\frac{\epsilon}{2} \\
& =\int_{R_{l, k}(0)}\left|w_{k}\right|^{2} d x d y+\frac{\epsilon}{2} \\
& \leq \int_{Q_{k}}\left|w_{k}\right|^{2} d x d y+\frac{\epsilon}{2} .
\end{aligned}
$$

Consequently,

$$
\int_{Q_{k}}\left|w_{k}\right|^{2} d x d y=\liminf _{n \rightarrow \infty} \int_{Q_{k}}\left|w_{n}\right|^{2} d x d y
$$

Now, using that $\left(\tilde{u}_{n}, \tilde{u}_{n}\right)_{n}$ is bounded in $\mathcal{X}_{k} \subset L^{q}\left(Q_{k}\right) \times \mathcal{M}^{q}\left(Q_{k}\right)$, that $\tilde{u}_{n} \rightarrow u_{k}$ and that $\partial_{i} \tilde{v}_{n} \rightarrow \partial_{i} v_{k}$ in $L^{2}\left(Q_{k}\right)$ for $i=1,2$, we conclude that

$$
\int_{Q_{k}}\left(\tilde{u}_{n}-u_{k}\right)\left(\partial_{i}\left(\tilde{v}_{n}-v_{k}\right)\right)^{p+1} d x d y=o(1), \quad \int_{Q_{k}}\left(\tilde{u}_{n}-u_{k}\right)\left(\partial_{x}\left(\tilde{v}_{n}-v_{k}\right)\right) d x d y=o(1)
$$

In other words, for $i=1,2$ we have that $G_{i, k}\left(\tilde{U}_{n}-U_{0, k}\right)=o(1)$. Moreover, a direct computation shows that

$$
G_{i, k}\left(\tilde{U}_{n}-U_{0, k}\right)=G_{i, k}\left(\tilde{U}_{n}\right)-G_{i, k}\left(U_{0, k}\right)+o(1) .
$$

But this implies that $G_{i, k}\left(U_{0, k}\right)=\lim _{n \rightarrow \infty} G_{i, k}\left(\tilde{U}_{n}\right)$. On the other hand, from the weak convergence on $X_{k}$ gives us that

$$
\Sigma_{k}\left(\tilde{U}_{n}\right)=\Sigma_{k}\left(\tilde{U}_{n}-U_{0, k}\right)+\Sigma_{k}\left(U_{0, k}\right)+o(1) .
$$

Since we have that

$$
0=\Lambda_{k}\left(\tilde{U}_{n}\right)=\Lambda_{k}\left(U_{0, k}\right)+2 \lim _{n \rightarrow \infty} \Sigma_{k}\left(\tilde{U}_{n}-U_{0, k}\right),
$$

we conclude that $\Lambda_{k}\left(U_{0, k}\right) \leq 0$. So, we have that $\mathcal{J}_{k}(c) \leq J_{c, k}\left(U_{0, k}\right)$ due to the characterization of the $\mathcal{J}_{k}(c)$. Moreover, we have that

$$
\begin{aligned}
J_{c, k}\left(U_{0, k}\right) \geq J_{k}(c) & =J_{c, k}\left(\tilde{U}_{n}\right)+o(1) \\
& =\Sigma_{k}\left(\tilde{U}_{n}-U_{0, k}\right)+\Sigma_{k}\left(U_{0, k}\right)+G_{2, k}\left(U_{0, k}\right)+o(1) \\
& \geq J_{c, k}\left(U_{0, k}\right)+o(1) .
\end{aligned}
$$

Note that this also proves that the subsequence $\left(\tilde{U}_{n}\right)_{n}$ converges to $U_{0, k}$ in $X_{k}$, since we already have that $\Lambda_{k}\left(U_{0, k}\right)=0$. In fact, suppose that $\Lambda_{k}\left(U_{0, k}\right)<0$. Then we can 
choose $\alpha \in(0,1)$ such that $\Lambda_{k}\left(\alpha U_{0, k}\right)=\alpha^{2}\left(2 \Sigma_{k}\left(U_{0, k}\right)+\alpha^{p}(p+2) G_{2, k}\left(U_{0, k}\right)\right)=0$. Thus we conclude that

$$
\mathcal{J}_{k}(c) \leq J_{c, k}\left(\alpha U_{0, k}\right)=\alpha^{2}\left(\frac{p}{p+2}\right) \Sigma_{k}\left(U_{0, k}\right)<\left(\frac{p}{p+2}\right) \Sigma_{k}\left(U_{0, k}\right)=\mathcal{J}_{k}(c) .
$$

This finishes the proof of this Lemma, since $\mathcal{J}_{k}(c)=\left(\frac{p}{p+2}\right) \Sigma\left(U_{0, k}\right)$ and $\Lambda_{k}\left(U_{0, k}\right)=0$, meaning that $U_{0, k}$ is a minimizer for $\mathcal{J}_{k}(c)=J_{c, k}\left(U_{0, k}\right)$.

Now we are in position to prove one of the main results in this section. We will see that the strong convergence is a consequence of the Lions's Concentration-Compactness Principle ([5]) applied to the nonnegative measure $\mu_{n}=$ $\left(\frac{p}{p+2}\right) \varrho\left(U_{n}\right) d V$, with density $\varrho$ defined by $(2.20)$.

Proposition 2.10. Let $0<|c|<1, \sigma>\frac{1}{2}$ and $k \geq 1$.

1. The family $\left(\mathcal{J}_{k}(c)\right)_{k \geq 1}$ is bounded below and above for positive constants independent of $k$.

2. If $\left\{U_{n}\right\}_{n}$ be a minimizing sequence for $\mathcal{J}_{k}(c)$. Then there exist a sequence of points $\zeta_{n} \in \mathbb{R}$ and a a subsequence of $\left\{U_{n}\right\}_{n}$ (denoted the same) and a minimizer $U_{0, k}$ for $\mathcal{J}_{k}(c)$ such that the translated sequence $\tilde{U}_{n}=U_{n}\left(\cdot+\left(0, \zeta_{n}\right)\right)$ converges strongly to $U_{0, k}$ in $X_{k}$.

Proof. 1.- The first observation is that there are positive constants $C_{i}=C_{i}(\sigma, c)$ for $i=1,2$ such that

$$
C_{1}\|U\|_{x_{k}}^{2} \leq \Sigma_{k}(U) \leq C_{2}\|U\|_{X_{k}}^{2}, \quad \text { for any } U \in X_{k} .
$$

Moreover, from (2.10) we have that

$$
\left|G_{2, k}(U)\right| \leq C(p)\|U\|_{x_{k}}^{p+2}, \quad \text { for any } U \in X_{k} .
$$

Let $\left\{V_{k}\right\}_{k} \in X_{k}$ be a family such that $J_{c, k}\left(V_{k}\right)=\mathcal{J}_{k}(c)$ with $\Lambda_{k}\left(V_{k}\right)=0$. Then we have that,

$$
\left(\frac{2 C_{1}}{p+2}\right)\left\|V_{k}\right\|_{\mathfrak{X}_{k}}^{2} \leq\left(\frac{2}{p+2}\right) \Sigma_{k}\left(V_{k}\right)=\left|G_{2, k}\left(V_{k}\right)\right| \leq C(p)\left\|V_{k}\right\|_{X_{k}}^{p+2},
$$

which implies that

$$
\left\|V_{k}\right\|_{X_{k}}^{2} \geq\left(\frac{2 C_{1}}{(p+2) C(p)}\right)^{2 / p}
$$

and so for the first inequality we conclude that

$$
\mathcal{J}_{k}(c)=\left(\frac{p}{p+2}\right) \Sigma_{k}\left(V_{k}\right) \geq C_{3}(c, \sigma, p) .
$$

On the other hand, for $k \geq 1$ we can choose $\varphi \in\left(C_{0}^{\infty}\left(Q_{1}\right)\right)^{2}$ having $G_{2,1}(\varphi)<0$. Since, $\operatorname{supp} \varphi \subset Q_{1} \subset Q_{k}$, we can define a periodic extension of $\varphi$ as follows

$$
\begin{array}{lll}
\varphi_{k}(x, y) & =\varphi(x, y), & (x, y) \in Q_{1}, \\
\varphi_{k}(x, y)=0, & (x, y) \in Q_{k} \backslash Q_{1} .
\end{array}
$$


We can take $\alpha \in \mathbb{R}$ in such a way that $e_{k}=\alpha \varphi_{k}$ satisfies that $e_{k} \in X_{k}$ and $\Lambda_{k}\left(e_{k}\right)=0$. Then, this allows us to conclude that

$$
\partial_{k}(c) \leq J_{c, k}\left(e_{k}\right)=J_{1}\left(e_{1}\right)=M_{4} .
$$

This means that there are positive constants $C_{4}, C_{5}$ (independent of $k$ ) such that

$$
C_{4} \leq \mathcal{J}_{k}(c) \leq C_{5}, \quad \text { for any } k \geq 1
$$

In particular, this allows to shows that $\mathcal{J}_{k}(c)>0$ for any $k \geq 1$.

2.- Let $k \geq 1$ be fixed and suppose that $\left\{U_{n}\right\}_{n}$ is a minimizing sequence for $\mathcal{J}_{k}(c)$. Then we have that $\left\|U_{n}\right\| x_{k}$ is a bounded sequence from bound (2.23), that $\Lambda_{k}\left(U_{n}\right)=0$ and

$$
\lim _{n \rightarrow \infty} J_{c, k}\left(U_{n}\right)=\lim _{n \rightarrow \infty}\left(\frac{p}{p+2}\right) \Sigma_{k}\left(U_{n}\right)=-\lim _{n \rightarrow \infty}\left(\frac{p}{2}\right) G_{2, k}\left(U_{n}\right)=\mathcal{J}_{k}(c)
$$

Using the variational characterization of $\mathcal{J}_{k}$ and the Concentration-Compactness Principle by P. Lion in [5], we have that the sequence of measure $\left(\mu_{n}\right)_{n}$ satisfies either Vanishing, Dichotomy or Compactness. Note that Lemma (2.5) implies that we do not have Vanishing.

We now claim that Dichotomy is not possible. In fact, assume that for some $\lambda \in$ $\left(0, \mathcal{J}_{k}(c)\right)$ there are a sequence of positive number $\left(r_{n}\right)_{n}$ tending to $\infty$ and a sequence $\left(\zeta_{n}\right)_{n} \subset \mathbb{R}$ with the following property: there are nonnegative measures $\mu_{n}^{1}, \mu_{n}^{2}$ such that

1. $0 \leq \mu_{n}^{1}+\mu_{n}^{2} \leq \mu_{n}$,

2. $\operatorname{supp}\left(\mu_{n}^{1}\right) \subset R_{r_{n}, k}\left(\zeta_{n}\right), \quad \operatorname{supp}\left(\mu_{n}^{2}\right) \subset Q_{k} \backslash R_{2 r_{n}, k}\left(\zeta_{n}\right)$,

3. $\lim \sup _{n \rightarrow \infty}\left(\left|\lambda-\int_{Q_{k}} d \mu_{n}^{1}\right|+\left|\left(\mathcal{J}_{k}(c)-\lambda\right)-\int_{Q_{k}} d \mu_{n}^{2}\right|\right)=0$.

Take a fixed $\phi \in C_{0}^{\infty}\left(\mathbb{R}, \mathbb{R}^{+}\right)$such that $\operatorname{supp}(\phi) \subset[-2,2]$ and $\phi \equiv 1$ in $[-1,1]$, and define

$$
\phi_{n}(y)=\phi\left(\frac{y-\zeta_{n}}{r_{n}}\right), \quad A(n)=R_{2 r_{n}, k}\left(\zeta_{n}\right) \backslash R_{r_{n}, k}\left(\zeta_{n}\right)
$$

Due to the nature of the space $X_{k}$ in the second component, we define the following non-standard splitting of $U_{n}=\left(u_{n}, v_{n}\right)$ :

$$
u_{n}^{1}=u_{n} \phi_{n}, \quad u_{n}^{2}=u_{n}\left(1-\phi_{n}\right), \quad v_{n}^{1}=\left(v_{n}-a_{n}\right) \phi_{n}, \quad v_{n}^{2}=\left(v_{n}-a_{n}\right)\left(1-\phi_{n}\right)+a_{n},
$$

where $a_{n}$ is given by

$$
a_{n}=\frac{1}{\operatorname{vol}\left(A_{n}\right)} \int_{A(n)} v_{n} d x d y
$$

As established in [4], we have the following properties for this non-standard splitting of $U_{n}$,

$$
\begin{aligned}
\Sigma_{k}\left(U_{n}\right) & =\Sigma_{k}\left(U_{n}^{1}\right)+\Sigma_{k}\left(U_{n}^{2}\right)+o(1), \\
G_{2, k}\left(U_{n}\right) & =G_{2, k}\left(U_{n}^{1}\right)+G_{2, k}\left(U_{n}^{2}\right)+o(1), \\
\Lambda_{k}\left(U_{n}\right) & =\Lambda_{k}\left(U_{n}^{1}\right)+\Lambda_{k}\left(U_{n}^{2}\right)+o(1) .
\end{aligned}
$$


Let $\lambda_{n, 1}=\Lambda_{k}\left(U_{n}^{1}\right)$ and $\lambda_{n, 2}=\Lambda_{k}\left(U_{n}^{2}\right)$. Suppose for example that $\lim _{n \rightarrow \infty} \lambda_{n, 1}>0$. Then we have that $\lim _{n \rightarrow \infty} \lambda_{n, 2}<0$. So, for $n$ large enough we have that $\lambda_{n, 2}^{n \rightarrow \infty}<0$. Thus, we can define $0 \leq \alpha_{n, 2} \leq 1$ as the solution of $\Lambda_{k}\left(\alpha_{n, 2} U_{n}^{2}\right)=0$. In other words, $\alpha_{n, 2}$ is the solution of the equation

$$
2 \Sigma_{k}\left(U_{n}^{2}\right)+(p+2) \alpha_{n, 2}^{p} G_{2, k}\left(U_{n}^{2}\right)=0 .
$$

Then from previous observation, we have that

$$
\begin{aligned}
\Sigma_{k}\left(U_{n}\right) & =\Sigma_{k}\left(U_{n}^{1}\right)+\Sigma_{k}\left(U_{n}^{2}\right)+\delta_{n} \\
& \geq \int_{R_{r_{n}, k}\left(\zeta_{n}\right)} \rho\left(U_{n}^{1}\right) d V+\alpha_{n, 2}^{-2} \Sigma_{k}\left(\alpha_{n, 2} U_{n}^{2}\right)+\delta_{n} \\
& \geq \int_{Q_{k}} d \mu_{n}^{1} d V+\alpha_{n, 2}^{-2}\left(\frac{p+2}{p}\right) \mathcal{J}_{k}(c)+\delta_{n} \\
& \geq \int_{Q_{k}} d \mu_{n}^{1} d V+\left(\frac{p+2}{p}\right) \mathcal{J}_{k}(c)+\delta_{n} .
\end{aligned}
$$

Taking limit as $n \rightarrow \infty$, we obtain the contradiction

$$
\mathcal{J}_{k}(c) \geq \lambda+\mathcal{J}_{k}(c)
$$

Now, we can assume for $i=1,2$ that $\lim _{n \rightarrow \infty} \lambda_{n, i}=0$. So, without loss of generality, we may take $U_{n} \neq 0$ for $n \in \mathbb{N}$. As above, for $i=1,2$ we define

$$
\alpha_{n, i}^{p}=-\frac{2 \Sigma_{k}\left(U_{n}^{i}\right)}{(p+2) G_{2, k}\left(U_{n}^{i}\right)}
$$

meaning that $\Lambda_{k}\left(U_{n}^{i}\right)=0$, and so

$$
\begin{aligned}
\Sigma_{k}\left(U_{n}\right) & =\alpha_{n, 1}^{-2} \Sigma_{k}\left(\alpha_{n, 1}^{2} U_{n}^{1}\right)+\alpha_{n, 2}^{-2} \Sigma_{k}\left(\alpha_{n, 2}^{2} U_{n}^{2}\right)+\delta_{n} \\
& \geq\left(\alpha_{n, 1}^{-2}+\alpha_{n, 2}^{-2}\right)\left(\frac{p+2}{p}\right) \mathcal{J}_{k}(c)+\delta_{n} .
\end{aligned}
$$

Since $\lim _{n \rightarrow \infty} \alpha_{n, i}=1$ for $i=1,2$, then we reach the contradiction $\mathcal{J}_{k}(c) \geq 2 \mathcal{J}_{k}(c)$, after taking the corresponding limit. In other words, we have ruled out Dichotomy. So, we have the Compactness property: there exists a sequence of points $\zeta_{n} \in \mathbb{R}$ such that for any $\epsilon>0$ there exists $l>0$ such that

$$
\left(\frac{p}{p+2}\right) \int_{R_{l, k}\left(\zeta_{n}\right)} \varrho\left(U_{n}\right) d x d y \geq \mathcal{J}_{k}(c)-\epsilon, \quad \text { for all } n \in \mathbb{N} .
$$

Thus, as a consequence of Lemma (2.9), we get the desired conclusion.

3. Interrelation between $x$-periodic travelling waves of period $k$ and solitons. From Theorem (2.7) or Theorem (2.10) we know the existence weak periodic travelling waves solution $U_{k}$ of the generalized Boussinesq system for $k \geq 1$, characterized as a minimizer for $\mathcal{J}_{k}(c)$ and also $U_{k}$ is a critical point of the functional $J_{c, k}$. We will see, after an appropriate translation, that there is a convergent subsequence in $X$ of the extended sequence $\left\{E_{k} U_{k}\right\}_{k}$, whose limit $U_{0}$ is a critical point of the functional $J_{c}$, with the property of being a weak solution of the Boussinesq 
system and a minimizer for $\mathcal{J}_{c}$ (a soliton (ground state) for the Boussinesq system). So, it is important in our analysis to recall that existence of solitary waves (solitions) was done by J. Quintero [1]. We want to point out that the proof of this result used the Mountain Pass Theorem. One of the main steps was to characterize the vanishing sequences. that

TheOrem 3.1. For $0<c<1$ and $\sigma>\frac{1}{2}$, there exists a non trivial $U_{0} \in \mathcal{X}$ such

$$
J_{c}\left(U_{0}\right)=\left(\frac{2^{\frac{2}{p}} p}{(p+2)^{\frac{p+2}{p}}}\right) \Upsilon(c)^{\frac{p+2}{p}}:=\mathcal{J}(c),
$$

where $\mathcal{J}(c)$ and $\Upsilon(c)$ are defined as

$$
\begin{aligned}
& \mathcal{J}(c)=\inf \left\{J_{c}(u): \Lambda(U)=0, U \in X \backslash\{0\}\right\}, \quad \Lambda(U)=\left\langle J^{\prime}(U), U\right\rangle \\
& \Upsilon(c)=\inf \left\{\Sigma(U): G_{2}(U)=1, \quad U \in \mathcal{X}\right\},
\end{aligned}
$$

with $J_{c}=\mathcal{J}+G_{1}+G_{2}, \Sigma=\mathcal{J}+G_{1}$ and the functionals $\mathcal{J}, G_{1}$ and $G_{2}$ are defined on X by

$$
\begin{aligned}
\mathcal{J}(u, v) & =\int_{\mathbb{R}^{2}}\left(u^{2}+\left(\sigma-\frac{1}{2}\right)|\nabla u|^{2}+|\nabla v|^{2}+\frac{1}{6}|\Delta v|^{2}\right) d y d x, \\
G_{1}(u, v) & =-2 c \int_{\mathbb{R}^{2}} u v_{x} d y d x \\
G_{2}(u, v) & =\frac{2}{p+1} \int_{\mathbb{R}^{2}} u\left(v_{x}^{p+1}+v_{y}^{p+1}\right) d y d x .
\end{aligned}
$$

From the same arguments used for the periodic space $x_{k}$ in $(2.11)$, we have the estimate

$$
C_{1}(\sigma, c)\|U\|_{X}^{2} \leq \Sigma(U) \leq C_{2}(\sigma, c)\|U\|_{X}^{2}
$$

Moreover, for $Q \subset \mathbb{R}^{2}$ we have a similar estimate

$$
C_{1}(\sigma, c)\|U\|_{\chi_{(Q)}}^{2} \leq \Sigma_{Q}(U) \leq C_{2}(\sigma, c)\|U\|_{X(Q)}^{2}
$$

where the integrals involved are taken on the set $Q$.

We observe that the key to establish the convergence of the sequence $\left\{E_{k} U_{k}\right\}_{k}$ is to prove the following result,

TheOREM 3.2. Let $R_{r}(\zeta)$ be a closed square centered at the point $\zeta \in \mathbb{R}^{2}$ of side $r$ and let $U_{k}=\left(u_{k}, v_{k}\right) \in X_{k}$ be a sequence of $x$-periodic functions of period $k$ such that $\left\|U_{k}\right\| x_{k} \leq C$. If there exists $r>0$ such that

$$
\lim _{k \rightarrow \infty}\left(\sup _{\zeta \in \mathbb{R}^{2}} \int_{R_{r}(\zeta)} \varrho_{1}\left(U_{k}\right) d x d y\right)=0 .
$$

Then, for any $q \geq 2$ we have that $\left\|\partial_{i} u_{k}\right\|_{L^{q}\left(Q_{k}\right)} \rightarrow 0$ and $\left\|v_{k}\right\|_{\mathcal{M}^{(q)}\left(Q_{k}\right)} \rightarrow 0$, for $i=1,2$.

The proof of this results is similar to the proof of Theorem (2.5). From this result, we are able to characterize the behavior of any bounded sequence $\left\{U_{k}\right\}_{k}$ satisfying that $J_{c, k}^{\prime}\left(U_{k}\right)=0$.

TheOREM 3.3. Let $U_{k} \in X_{k}$ be a sequence of $x$-periodic functions of period $k$ such that $\left\|U_{k}\right\| x_{k} \leq C$ and $J_{c, k}^{\prime}\left(U_{k}\right)=0$. then either 
1. $\lim _{k \rightarrow \infty}\left\|U_{k}\right\| x_{k}=0$, or

2. there are positive constants $r, \eta$, and a sequence $\zeta_{k} \in \mathbb{R}^{2}$ such that, along a subsequence

$$
\lim _{k \rightarrow \infty}\left(\int_{R_{r}\left(\zeta_{k}\right)} \varrho_{1}\left(U_{k}\right) d x d y\right)>\eta
$$

Proof. Note that $J_{c, k}^{\prime}\left(U_{k}\right)\left(U_{k}\right)=2 \Sigma_{k}\left(U_{k}\right)+(p+2) G_{2, k}\left(U_{k}\right)=0$. Then, from estimates (2.10) and (2.24) we conclude for $U_{k}=\left(u_{k}, v_{k}\right)$ that

$$
\left\|U_{k}\right\|_{\mathfrak{X}_{k}}^{2} \leq C_{1}^{-1} \Sigma_{k}\left(U_{k}\right) \leq C_{6}\left(\left\|\left.\partial_{i} u_{k}\right|_{L^{p+2}\left(Q_{k}\right)} ^{p+2}+\right\| v_{k} \|_{\mathcal{M}\left(Q_{k}\right)^{p+2}}^{p+2}\right) .
$$

In case (2) does not hold, then from previous result we conclude that the right hand side tends to zero, which implies that condition (1) holds.

Now, we have the main result in this section,

THEOREM 3.4. Let $U_{k} \in \mathcal{X}_{k}$ be a minimizer for $\mathcal{J}_{k}(c)$. Then there exists a sequence $\zeta_{k} \in \mathbb{R}^{2}$ and a function $U_{0} \in X$ such that $E_{k} U_{k}\left(\cdot+\zeta_{k}\right)$ converges weakly to $U_{0}$ along a subsequence. Moreover, $U_{0}$ is a nontrivial solution of the Boussinesq system, a minimizer for $\mathcal{J}$, and

$$
\lim _{k \rightarrow \infty}\left\|U_{k}-U_{0}\left(\cdot-\zeta_{k}\right)\right\| x_{k}=0 .
$$

Proof. By bounds (2.23) and (2.24) Proposition (2.10) we have for constants $C_{1}, C_{2}, C_{4}, C_{5}$ independent of $k$ that

$$
C_{1}\left\|U_{k}\right\|_{x_{k}}^{2} \leq \Sigma_{k}\left(U_{k}\right) \leq C_{2}\left\|U_{k}\right\|_{x_{k}}^{2} \quad \text { and } \quad 0<C_{4} \leq J_{c, k}\left(U_{k}\right) \leq C_{5}
$$

Recall that we have $J_{c, k}\left(U_{k}\right)=\left(\frac{p}{p+2}\right) \Sigma_{k}\left(U_{k}\right)$, so we can conclude for constants $C_{6}, C_{7}$ independent of $k$ that

$$
0<C_{6}(p) \leq\left\|U_{k}\right\| x_{k} \leq C_{7}(p),
$$

implying that condition (1) in Theorem (3.3) does not hold. In other words, there exists a sequence $\zeta_{k} \in \mathbb{R}^{2}$ such that the shifted sequence $\tilde{U}_{k}=U_{k}\left(\cdot+\zeta_{k}\right)$, for appropriate choice of $r, \eta>0$, satisfies

$$
\int_{R_{r}(0)} \varrho_{1}\left(\tilde{U}_{k}\right) d x d y>\frac{\eta}{2} .
$$

Clearly, $\tilde{U}_{k}$ is also a minimizer for $\mathcal{J}_{k}$, which means that

$$
J_{c, k}^{\prime}\left(\tilde{U}_{k}\right)(\phi)=\Sigma_{k}^{\prime}\left(\tilde{U}_{k}\right)(\phi)+G_{2, k}^{\prime}\left(\tilde{U}_{k}\right)(\phi)=0, \text { for all } \phi \in X_{k} .
$$

Now, we observe that the sequence $\left\{E_{k} \tilde{U}_{k}\right\}_{k} \subset X$ is bounded since

$$
\left\|E_{k} \tilde{U}_{k}\right\|_{x} \leq D_{3}\left\|\tilde{U}_{k}\right\|_{x_{k}}
$$

Then there exists a subsequence of $\left\{E_{k} \tilde{U}_{k}\right\}_{k}$ (denoted the same) and $U_{0} \in X$ such that

$$
E_{k} \tilde{U}_{k} \rightarrow U_{0} \quad(\text { weakly in } X)
$$


Using the uniform estimate (3.28) and that the embedding $X \hookrightarrow L_{l o c}^{q}\left(\mathbb{R}^{2}\right) \times \mathcal{M}_{l o c}^{(q)}\left(\mathbb{R}^{2}\right)$ is compact for $q \geq 2$ (see Lemma (2.1)), we are able to see that $U_{0} \neq 0$. In fact, we can assume that $\left\{E_{k} \tilde{U}_{k}\right\}_{k}$ converges strongly to $U_{0}$ in $L_{l o c}^{q}(Q) \times \mathcal{M}_{l o c}^{(q)}(Q)$. So, for $q=2$ we have from (3.27) that

$$
C_{2}(\sigma, c)\left\|U_{0}\right\|_{X\left(R_{r}(0)\right)}^{2} \geq \int_{R_{r}(0)} \varrho_{1}\left(U_{0}\right) d x d y=\lim _{k \rightarrow \infty} \int_{R_{r}(0)} \varrho_{1}\left(\tilde{U}_{k}\right) d x d y \geq \frac{\delta^{*}}{2} .
$$

In order to see that $U_{0}$ is a weak nontrivial solution of the Boussinesq system (1.1), we will show that

$$
J_{c}^{\prime}\left(U_{0}\right)(\phi)=\Sigma^{\prime}\left(U_{0}\right)(\phi)+G_{2, c}^{\prime}\left(U_{0}\right)(\phi)=0 \text { for all } \phi \in X .
$$

If we consider $\phi \in C_{0}^{\infty}\left(\mathbb{R}^{2}\right)$, then for sufficiently large $k$ we have that $\operatorname{supp} \phi=\Omega \subset Q_{k}$. So, $\phi$ can be considered as an element of either $\mathcal{V}_{k}$ or $H_{k}^{1}$, for $k$ large just by defining its periodic extension. Now, for $\tilde{U}_{k}=\left(\tilde{u}_{k}, \tilde{v}_{k}\right)$ and $\phi=(z, w) \in\left(C_{0}^{\infty}\left(\mathbb{R}^{2}\right)\right)^{2}$ with $\operatorname{supp} \phi \subset Q_{k}$, we have that

$$
\begin{aligned}
\Sigma^{\prime}\left(E_{k} \tilde{U}_{k}\right)(\phi)= & \int_{\mathbb{R}^{2}} F_{k} \tilde{u}_{k} z+\left(\sigma-\frac{1}{2}\right)\left(\partial_{x} F_{k} \tilde{u}_{k} z_{x}+\partial_{y} F_{k} \tilde{u}_{k} z_{y}\right)+\left(\partial_{x} S_{k} \tilde{v}_{k} w_{x}+\partial_{y} S_{k} \tilde{v}_{k} w_{y}\right) \\
& +\frac{1}{6} \Delta S_{k} \tilde{v}_{k} \Delta w-c z \partial_{x} \tilde{v}_{k}-c \tilde{u}_{k} w_{x} d x d y \\
= & \int_{\Omega} \tilde{u}_{k} z+\left(\sigma-\frac{1}{2}\right)\left(\partial_{x} \tilde{u}_{k} z_{x}+\partial_{y} \tilde{u}_{k} z_{y}\right)+\left(\partial_{x} \tilde{v}_{k} w_{x}+\partial_{y} \tilde{v}_{k} w_{y}\right) \\
& +\frac{1}{6} \Delta \tilde{v}_{k} \Delta w-c z \partial_{x} \tilde{v}_{k}-c \tilde{u}_{k} w_{x} d x d y \\
= & \int_{Q_{k}} \tilde{u}_{k} z+\left(\sigma-\frac{1}{2}\right)\left(\partial_{x} \tilde{u}_{k} z_{x}+\partial_{y} \tilde{u}_{k} z_{y}\right)+\left(\partial_{x} \tilde{v}_{k} w_{x}+\partial_{y} \tilde{v}_{k} w_{y}\right) \\
& +\frac{1}{6} \Delta \tilde{v}_{k} \Delta w-c z \partial_{x} \tilde{v}_{k}-c \tilde{u}_{k} w_{x} d x d y \\
= & \Sigma_{k}^{\prime}\left(\tilde{U}_{k}\right)(\phi),
\end{aligned}
$$

which clearly implies that $\Sigma_{k}^{\prime}\left(\tilde{U}_{k}\right)(\phi) \rightarrow \Sigma^{\prime}\left(\tilde{U}_{0}\right)(\phi)$, since for $k$ large enough $\phi$ can be considered in the space $X_{k}$. On the other hand, we know that

$$
\left\{\left(\partial_{i} \tilde{v}_{k}\right)^{p+1}\right\}_{k} \text { and }\left\{\tilde{u}_{k}\left(\partial_{y} \tilde{v}_{k}\right)^{p}\right\}_{k}
$$

are bounded in $L^{\frac{p+2}{p+1}}\left(Q_{k}\right)$. This fact allows us to conclude that

$$
G_{2, k}^{\prime}\left(\tilde{U}_{k}\right)(\phi)=G_{2}^{\prime}\left(E_{k} \tilde{U}_{k}\right)(\phi) \rightarrow G_{2}^{\prime}\left(U_{0}\right)(\phi) .
$$

As a consequence of this, we have that

$$
\begin{aligned}
J_{c}^{\prime}\left(U_{0}\right)(\phi) & =\Sigma^{\prime}\left(U_{0}\right)(\phi)+G_{2, c}^{\prime}\left(U_{0}\right)(\phi) \\
& =\lim _{k \rightarrow \infty}\left(\Sigma_{k}^{\prime}\left(\tilde{U}_{k}\right)(\phi)+G_{2, k}^{\prime}\left(\tilde{U}_{k}\right)(\phi)\right) \\
& =\lim _{k \rightarrow \infty} J_{c, k}^{\prime}\left(\tilde{U}_{k}\right)(\phi)=0 .
\end{aligned}
$$

In other words, $U_{0}$ is a weak nontrivial solution of the Boussinesq system (1.1). Finally we want to show that $J_{c}\left(U_{0}\right)=\mathcal{J}(c)=\{U \in \mathcal{X} \backslash\{0\}: \Lambda(U)=0\}$ and that $\| \tilde{U}_{k}-$ 
$U_{0} \| x_{k} \rightarrow 0$ as $k \rightarrow \infty$. First note that by a density argument for given $W \in X$ such that $\Lambda(W)=0$, there exists a sequence $W_{k} \in\left(C_{0}^{\infty}\left(Q_{k}\right)\right)^{2}$ such that

$$
\left\|W_{k}-W\right\|_{x} \rightarrow 0, \quad \text { as } k \rightarrow \infty .
$$

By the assumption on $W$, it follows that $G_{2}(W)<0$. By continuity of functional $G_{2}$, we have for $k$ large enough that $G_{2}\left(W_{k}\right)<0$. Moreover, for $t_{k}^{p}=-\left(\frac{2}{p+2}\right) \frac{\Sigma\left(W_{k}\right)}{G_{2}\left(W_{k}\right)}$, we have that $t_{k} \in(0,1)$ and that $\Lambda\left(t_{k} W_{k}\right)=0$. Note that the continuity of functionals $\Sigma$ and $G_{2}$, and that $\Lambda(W)=0$ imply that

$$
t_{k}=\left(-\left(\frac{2}{p+2}\right) \frac{\Sigma\left(W_{k}\right)}{G_{2}\left(W_{k}\right)}\right)^{1 / p} \rightarrow\left(-\left(\frac{2}{p+2}\right) \frac{\Sigma(W)}{G_{2}(W)}\right)^{1 / p}=1, \text { as } k \rightarrow \infty .
$$

On the other hand, $Z_{k}=t_{k} W_{k} \rightarrow W$ in $X$. Then from the continuity of the functional $J_{c}$, we conclude that $J_{c}\left(Z_{k}\right) \rightarrow J_{c}(W)$, as $k \rightarrow \infty$. Thus given $\epsilon>0$, there exists $k_{\epsilon}$ such that for $k \geq k_{\epsilon}$

$$
J_{c}\left(Z_{k}\right) \leq J_{c}(W)+\epsilon
$$

which implies that $\lim \sup \mathcal{J}_{k}(c) \leq J(W)+\epsilon$ for any $W \in \mathcal{X}$ with $\Lambda(W)=0$, and for any $\epsilon>0$. Moreover,

$$
\lim \sup \mathcal{J}_{k}(c) \leq \mathcal{J}(c)
$$

Now, we have to recall that

$$
\jmath_{k}(c)=J_{c, k}\left(\tilde{U}_{k}\right)=\left(\frac{p}{p+2}\right) \Sigma_{k}\left(\tilde{U}_{k}\right)=\left(\frac{p}{p+2}\right) \int_{Q_{k}} \varrho\left(\tilde{U}_{k}\right) d x d y .
$$

Note that for a given bounded domain $\Omega \subset \mathbb{R}^{2}$, we have that $\Omega \subset Q_{k}$ for $k$ large enough, and so,

$$
\mathcal{J}_{k}(c)=J_{c, k}\left(\tilde{U}_{k}\right) \geq\left(\frac{p}{p+2}\right) \int_{\Omega} \varrho\left(\tilde{U}_{k}\right) d x d y
$$

Taking liminf we get that

$$
\liminf \mathcal{J}_{k}(c) \geq\left(\frac{p}{p+2}\right) \liminf \int_{\Omega} \varrho\left(\tilde{U}_{k}\right) d x d y \geq\left(\frac{p}{p+2}\right) \int_{\Omega} \varrho\left(U_{0}\right),
$$

due to the local compactness result. In other words, we have shown that

$$
\liminf \mathcal{J}_{k}(c) \geq\left(\frac{p}{p+2}\right) \int_{\mathbb{R}^{2}} \varrho\left(U_{0}\right)=\left(\frac{p}{p+2}\right) \Sigma\left(U_{0}\right),
$$

since $\Omega$ is arbitrary. But, $\Lambda\left(U_{0}\right)=0$ since $U_{0}$ is a non trivial weak solution of the Boussinesq system ((1.1). Then,

$$
\liminf \mathcal{J}_{k}(c) \geq\left(\frac{p}{p+2}\right) \int_{\mathbb{R}^{2}} \varrho\left(U_{0}\right)=\left(\frac{p}{p+2}\right) \Sigma\left(U_{0}\right) \geq \mathcal{J}(c) .
$$

In other words,

$$
\lim _{k \rightarrow \infty} \mathcal{J}_{k}(c)=\mathcal{J}(c)=\left(\frac{p}{p+2}\right) \Sigma\left(U_{0}\right)=J_{c}\left(U_{0}\right)
$$


which is equivalent to say that $U_{0}$ is a ground state. Note that we also proved that

$$
\lim \Sigma_{k}\left(\tilde{U}_{k}\right)=\Sigma\left(U_{0}\right)
$$

It remains to prove that

$$
\lim _{k \rightarrow \infty}\left\|U_{k}-U_{0}\left(\cdot-\zeta_{k}\right)\right\|_{x_{k}}=0 .
$$

To see this, let $W_{k} \in\left(C_{0}^{\infty}\left(Q_{k}\right)\right)^{2}$ such that $W_{k} \rightarrow U_{0}$ in $X$. Then a direct computation shows that

$$
\lim _{k \rightarrow \infty}\left\|U_{k}-U_{0}\left(\cdot-\zeta_{k}\right)\right\| x_{k}=\lim _{k \rightarrow \infty}\left\|\tilde{U}_{k}-U_{0}\right\|_{x_{k}}=0 \Leftrightarrow \lim _{k \rightarrow \infty}\left\|\tilde{U}_{k}-W_{k}\right\|_{x_{k}}=0 .
$$

Now,

$$
\begin{aligned}
\Sigma_{k}\left(\tilde{U}_{k}-W_{k}\right) & =\Sigma_{k}\left(\tilde{U}_{k}\right)+\Sigma_{k}\left(W_{k}\right)-2 \Sigma_{k}^{\prime}\left(\tilde{U}_{k}\right)\left(W_{k}\right) \\
& =\Sigma_{k}\left(\tilde{U}_{k}\right)+\Sigma_{k}\left(W_{k}\right)-\Sigma_{k}^{\prime}\left(\tilde{U}_{k}\right)\left(U_{0}\right)-\Sigma_{k}^{\prime}\left(\tilde{U}_{k}\right)\left(W_{k}-U_{0}\right) .
\end{aligned}
$$

Since $W_{k}$ converges strongly to $U_{0}$ in $\mathcal{X}$ and $\left\|\tilde{U}_{k}\right\|_{x_{k}}$ is bounded, we conclude that

$$
\left|\Sigma_{k}^{\prime}\left(\tilde{U}_{k}\right)\left(W_{k}-U_{0}\right)\right| \leq C(c)\left\|\tilde{U}_{k}\right\|_{x_{k}}\left\|W_{k}-U_{0}\right\|_{x}=o(1) .
$$

But we proved that $\Sigma_{k}^{\prime}\left(\tilde{U}_{k}\right)\left(U_{0}\right) \rightarrow \Sigma_{k}^{\prime}\left(\tilde{U}_{0}\right)\left(U_{0}\right)=2 \Sigma_{k}\left(U_{0}\right)$. So, taking limit as $k \rightarrow \infty$ and using the estimate $(2.23)$,

$$
\left\|\tilde{U}_{k}-W_{k}\right\|_{\Upsilon_{k}}^{2} \sim \Sigma_{k}\left(\tilde{U}_{k}-W_{k}\right)=o(1) .
$$

As desired,

$$
\lim _{k \rightarrow \infty}\left\|U_{k}-U_{0}\left(\cdot-\zeta_{k}\right)\right\|_{x_{k}}=0
$$

Acknowledgments. JRQ was supported by Universidad del Valle and the Mathematics Department under the project C.I. 7910.

\section{REFERENCES}

[1] J. R. Quintero, Solitary water waves for a 2D Boussinesq Type System, J. Partial Differential Equations, 23 (2010), pp. 251-280.

[2] A. Pankov And K. Pflüger, Periodic and Solitary Waves Solutions for the Generalized Kadomtsev-Petviashvili Equation, Mathematical Methods in the Applied Science, 22:9 (1999), pp. 733-752.

[3] J. R. Quintero, The Cauchy problem and stability of solitary waves for a 2D Boussinesq -KdV Type System, J. Differential and Integral Equations, 24 (2011), pp. 325-360.

[4] J. R. Quintero, Solitons and periodic travelling waves for the 2D-generalized Benney-Luke equation, Applicable Analysis, 86 (2007), pp. 331-351.

[5] P. L. Lions, The Concentration Compactness Method in the Calculus of variations, The Locally Compact Case.I, II, Partial Differential Equations and Hamiltonian Systems, Ann. Inst, H. Poinacré, Anal Non Linéaire, 1 (1984), pp. 109-145, pp. 223-283.

[6] J. Bona, M. Chen, AND J.-C. SAUt, Boussinesq equations and other systems for smallamplitude long waves in nonlinear dispersive media I: Derivation and the linear theory, J. Nonlinear Sci., 12 (2002), pp. 283-318.

[7] J. Bona, M. Chen, And J.-C. SAUt, Boussinesq equations and other systems for smallamplitude long waves in nonlinear dispersive media II: Nonlinear theory, Nonlinearity, 17 (2004), pp. 925-952. 
[8] J. Bona, T. Colin, And D. LAnnes, Long wave approximations for water waves, Arch. Rational Mech. Anal., 178 (2005), pp. 373-410.

[9] M. D. Groves, Three-dimensional travelling gravity-capillary water waves, GAMM-Mitt. 30, 30:1 (2007), pp. 8-43.

[10] D. Lannes, The water waves problem- Mathematical Analysis and Asymptotics, Mathematical Surveys and Monographs, 188. AMS, Providence, RI., 2013.

[11] M. Chen, N. NGuyen, And S. Sun, Solitary-wave solutions to Boussinesq systems with large surface tension, Dis. Cont. Dyna. Sys. A., 26 (2010), pp. 1153-1184.

[12] M. Chen, N. NGuyen, And S. Sun, Existence of travelling waves solutions to Boussinesq systems, Differential and Integral equations, 24 (2011), pp. 895-908.

[13] J. R. Quintero And J. Angulo, Existence and Orbital Stability of Cnoidal Waves for a 1D Boussinesq equation, International Journal of Mathematics and Mathematical Science, ID 52020 (2007).

[14] J. Angulo, Nonlinear Dispersive Equations. Existence and Stability of Solitary and Perioidic Travelling Waves Solutions, SURV Applied Mathematics, 156, AMS, 2009.

[15] J. Angulo, Stability of cnoidal waves to Hirota-Satsuma systems, Matemática Contemporânea, 27 (2004), pp. 189-223.

[16] M. Willem, Minimax Methods, Boston: Birkhäuser (1996).

[17] A. Ambrosetti, P. H. Rabinowitz, Dual variational methods in critical point theory and applications, J. Funct. Ana., 14 (1973), pp. 349-381.

[18] R. L. Pego And J. Quintero, Two-dimensional solitary Waves for a Benney-Luke equation, Phisica D, 45 (1999), pp. 476-496.

[19] D. J. Benney And J. C. Luke, Interactions of permanent waves of finite amplitude, J. Math. Phys., 43 (1964), pp. 309-313.

[20] A. PAZY, Semigroups of linear operators and applications. Appl. Math. Sci., volume 44. Springer-Verlag, NY, 1983. 